\title{
DETECTION OF REST-FRAME OPTICAL LINES FROM X-SHOOTER SPECTROSCOPY OF WEAK EMISSION-LINE QUASARS*
}

\author{
Richard M. Plotkin $^{1}$, Ohad Shemmer ${ }^{2}$, Benny Trakhtenbrot ${ }^{3,13}$, Scott F. Anderson ${ }^{4}$, W. N. Brandt ${ }^{5,6,7}$, Xiaohui Fan $^{8}$, \\ Elena Gallo ${ }^{1}$, Paulina Lira ${ }^{9}$, Bin Luo ${ }^{5,6}$, Gordon T. Richards ${ }^{10}$, Donald P. Schneider ${ }^{5,6}$, Michael A. Strauss ${ }^{11}$, and \\ JIANFENG Wu ${ }^{12}$ \\ ${ }^{1}$ Department of Astronomy, University of Michigan, 1085 South University Avenue, Ann Arbor, MI 48109, USA; rplotkin@umich.edu \\ ${ }_{2}^{2}$ Department of Physics, University of North Texas, Denton, TX 76203, USA \\ ${ }^{3}$ Institute for Astronomy, Department of Physics, ETH Zurich, Wolfgang-Pauli-Strasse 27, CH-8093 Zurich, Switzerland \\ ${ }^{4}$ Department of Astronomy, University of Washington, Box 351580, Seattle, WA 98195, USA \\ ${ }^{5}$ Department of Astronomy \& Astrophysics, 525 Davey Lab, The Pennsylvania State University, University Park, PA 16802, USA \\ ${ }_{7}^{6}$ Institute for Gravitation and the Cosmos, The Pennsylvania State University, University Park, PA 16802, USA \\ ${ }^{7}$ Department of Physics, 104 Davey Lab, The Pennsylvania State University, University Park, PA 16802, USA \\ ${ }^{8}$ Steward Observatory, University of Arizona, 933 North Cherry Avenue, Tucson, AZ 85721, USA \\ ${ }^{9}$ Departamento de Astronomia, Universidad de Chile, Camino del Observatorio 1515, Santiago, Chile \\ ${ }^{10}$ Department of Physics, Drexel University, 3141 Chestnut Street, Philadelphia, PA 19104, USA \\ ${ }^{11}$ Department of Astrophysical Sciences, Princeton University, Princeton, NJ 08544, USA \\ ${ }^{12}$ Harvard-Smithsonian Center for Astrophysics, MS 6, 60 Garden Street Cambridge, MA 02138, USA \\ Received 2015 January 20; accepted 2015 March 22; published 2015 May 28
}

\begin{abstract}
Over the past $15 \mathrm{yr}$, examples of exotic radio-quiet quasars with intrinsically weak or absent broad emission line regions (BELRs) have emerged from large-scale spectroscopic sky surveys. Here, we present spectroscopy of seven such weak emission line quasars (WLQs) at moderate redshifts $(z=1.4-1.7)$ using the X-shooter spectrograph, which provides simultaneous optical and near-infrared spectroscopy covering the rest-frame ultraviolet (UV) through optical. These new observations effectively double the number of WLQs with spectroscopy in the optical rest-frame, and they allow us to compare the strengths of (weak) high-ionization emission lines (e.g., $\mathrm{C}$ IV) to low-ionization lines (e.g., $\mathrm{Mg} \mathrm{II}, \mathrm{H} \beta, \mathrm{H} \alpha$ ) in individual objects. We detect broad $\mathrm{H} \beta$ and $\mathrm{H} \alpha$ emission in all objects, and these lines are generally toward the weaker end of the distribution expected for typical quasars (e.g., $\mathrm{H} \beta$ has rest-frame equivalent widths ranging from 15-40 $\AA$ ). However, these lowionization lines are not exceptionally weak, as is the case for high-ionization lines in WLQs. The X-shooter spectra also display relatively strong optical Fe II emission, $\mathrm{H} \beta \mathrm{FWHM} \lesssim 4000 \mathrm{~km} \mathrm{~s}^{-1}$, and significant C IV blueshifts $\left(\approx 1000-5500 \mathrm{~km} \mathrm{~s}^{-1}\right)$ relative to the systemic redshift; two spectra also show elevated UV Fe II emission, and an outflowing component to their (weak) Mg II emission lines. These properties suggest that WLQs are exotic versions of "wind-dominated" quasars. Their BELRs either have unusual high-ionization components, or their BELRs are in an atypical photoionization state because of an unusually soft continuum.
\end{abstract}

Key words: accretion, accretion disks - galaxies: active - quasars: emission lines

\section{INTRODUCTION}

One of the prominent observational signatures of Type 1 quasars is radiation from the broad emission line region (BELR). The BELR is composed of high-velocity gas $\left(\gtrsim 10^{3} \mathrm{~km} \mathrm{~s}^{-1}\right)$ embedded deep in the gravitational potential well of a quasar's supermassive black hole (SMBH; $\lesssim 0.1 \mathrm{pc}$ ), which reprocesses photons from the accretion disk and X-ray corona into Doppler broadened line emission. The BELR responds to variations of the quasar's central engine (e.g., Peterson 1993), and its properties (e.g., differences in the relative strengths and widths of various emission species, nonvirialized motions, etc.) are influenced by fundamental quasar parameters like luminosity, black hole mass $\left(M_{\mathrm{BH}}\right)$, and Eddington ratio $\left(L_{\mathrm{bol}} / \mathrm{L}_{\mathrm{Edd}}\right)^{14}$ (e.g., Baldwin 1977; Boroson \& Green 1992; Murray et al. 1995; Murray \& Chiang 1995; Elvis 2000; Nicastro 2000; Sulentic et al. 2007; Elitzur \&

\footnotetext{
* Based on observations made with ESO Telescopes at the La Silla Paranal Observatory under program IDs 088.B-0355 and 090.B-0438.

13 Zwicky Postdoctoral Fellow.

${ }^{14} L_{\text {bol }}$ is the bolometric luminosity, and $L_{\mathrm{Edd}}=1.26 \times 10^{38}\left[M_{\mathrm{BH}} / M_{\odot}\right]$ $\mathrm{erg} \mathrm{s}^{-1}$ is the Eddington luminosity for ionized hydrogen in a spherical geometry.
}

Ho 2009; Richards et al. 2011; Shen \& Ho 2014). The BELR is therefore a powerful tool for constraining the energetics of accreting black holes and for probing the link between SMBHs and their host galaxies.

An unexpected population of $\sim 10^{2}$ quasars that display exceptionally weak or completely missing broad emission lines in the ultraviolet (UV) rest-frame (Fan et al. 1999; Anderson et al. 2001; Collinge et al. 2005; Diamond-Stanic et al. 2009; hereafter DS09; Plotkin et al. 2010a; hereafter P10; Hryniewicz et al. 2010; Wu et al. 2011; Meusinger \& Balafkan 2014) was discovered within the spectra of the Sloan Digital Sky Survey (SDSS; York et al. 2000). The first systematic search for such weak emission line quasars (WLQs) was performed by DS09, who examined the rest-frame equivalent width $\left(W_{r}\right)$ distribution of the $\operatorname{Ly} \alpha \lambda 1216+\mathrm{N} v \lambda 1240$ blend for SDSS quasars at $z>3$. They identified $\sim 70$ WLQs, defined by an excess in the number of high-redshift SDSS quasars with $W_{r}[\mathrm{Ly} \alpha$ $+\mathrm{N} \mathrm{v}]<15 \AA$, which corresponds to the $>3 \sigma$ weak tail of the $W_{r}[\mathrm{Ly} \alpha+\mathrm{N} \mathrm{v}]$ distribution. A comparable number of lowerredshift $(z<3)$ WLQs have subsequently been identified within the SDSS as well, with the majority being discovered as a byproduct of searches for BL Lac objects (Collinge et al. 2005; $\mathrm{P} 10)$, as well as through machine learning data mining 
techniques (Meusinger \& Balafkan 2014) and serendipitous discoveries (Hryniewicz et al. 2010; Nikołajuk \& Walter 2012). Most of the $z<3$ WLQs are at too low-redshift for the Lya $+\mathrm{N} v$ blend to be covered by the SDSS spectrograph, and they are generally selected by weak $\operatorname{Mg}$ II $\left.\lambda 2800, \mathrm{C}_{\text {III }}\right] \lambda 1909$, and/ or $\mathrm{C}$ IV $\lambda 1549$ (depending on the redshift; see above references for details).

The puzzle behind WLQs is that they appear to be typical quasars in almost every aspect except for their unusually weak broad emission lines. The only property that may hint at a difference from the parent quasar population is that about half of all WLQs are X-ray weaker than expected given their restframe UV luminosities (Shemmer et al. 2009; Wu et al. 2011, 2012; Luo et al. 2015). Otherwise, WLQ optical colors and variability amplitudes/timescales are similar to other Type 1 SDSS quasars, showing little to no evidence of significant absorption or obscuration by the dusty torus (Fan et al. 1999; DS09). Dilution of emission lines by a beamed relativistic jet (as is partly responsible for the weak BELR emission from BL Lac objects; Blandford \& Rees 1978, pp. 328-41) is also extremely unlikely: WLQs are not radio-loud ${ }^{15}$ (Collinge et al. 2005; DS09; P10), their optical emission is not polarized (Fan et al. 1999; Smith et al. 2007; DS09; Heidt \& Nilsson 2011), their X-ray to optical luminosity ratios are smaller than for BL Lac objects (Shemmer et al. 2009; P10; Plotkin et al. 2010b; Wu et al. 2011, 2012), and their infrared (IR) colors are also different from BL Lac objects (Plotkin et al. 2012). Further arguing against a beamed synchrotron continuum, the UV through IR spectral energy distributions (SEDs) of WLQs are similar to other SDSS quasars, showing a "big blue bump" emission in the UV and reprocessed dust radiation in the IR (Lane et al. 2011; Wu et al. 2012). WLQs are also far too luminous for their weak or absent BELRs to be explained by a radiatively inefficient accretion flow due to low Eddington ratios, as has been proposed for so-called "optically dull" active galactic nuclei (AGNs; e.g., Nicastro et al. 2003; Hawkins 2004; Tran et al. 2011; Trump et al. 2011). Finally, there is no evidence that effects from gravitational lensing are important for the weak BELR emission from WLQs (Shemmer et al. 2006; DS09).

Considering the above, WLQs represent one extreme of quasar physics that is driven predominantly by intrinsic properties of the quasar, and not only by orientation. Several models have been proposed to explain this extreme population. These models fall into two broad categories: (i) WLQ BELRs are unusually gas deficient (we refer to this as the anemic BELR idea) and (ii) WLQ BELRs are in an unusual ionization state, most likely caused by a softer than normal ionizing continuum (which we refer to as the soft continuum idea). For the anemic category, WLQs could simply have low gas content and/or covering factors (e.g., Shemmer et al. 2010; Nikołajuk $\&$ Walter 2012). In this case, we may expect all broad lines to have low equivalent widths and luminosities. Another potential explanation within the anemic category is that WLQs are just beginning a quasar phase, and their BELRs are bare because a disk wind has not yet had sufficient time to lift material from the accretion disk into the BELR (Hryniewicz et al. 2010). Assuming disk wind velocities of $\approx 10^{2} \mathrm{~km} \mathrm{~s}^{-1}$, Hryniewicz

\footnotetext{
${ }^{15}$ Radio loudness is typically determined by the parameter $R=f_{5 \mathrm{GHz}} / f_{4400} \AA$, where $f_{5 \mathrm{GHz}}$ and $f_{4400} \AA$ are radio and optical flux densities at $5 \mathrm{GHz}$ and $4400 \AA$, respectively. Radio-loud quasars are usually defined by $R>10-100$ (e.g., Kellermann et al. 1989).
}

et al. (2010) propose that BELR formation should take $\sim 10^{3} \mathrm{yr}$ and WLQs should therefore be rare. During the WLQ phase, low-ionization emission lines that form close to the accretion disk (e.g., $\mathrm{H} \beta, \mathrm{Mg}$ II) may appear relatively normal, while higher-ionization lines with "wind" components (e.g., C IV) appear weakest because they originate in a region higher above the disk that has not yet fully formed.

The soft continuum category can be subdivided into physical mechanisms that produce intrinsically soft continua, and those that modify the continuum prior to illuminating the BELR (e.g., through absorption). One way to produce an intrinsically soft continuum is through a cold accretion disk. Laor \& Davis (2011) show that, since the temperature of a standard thin accretion disk scales as $M_{\mathrm{BH}}^{0.25}$, extremely massive SMBHs $\left(M_{\mathrm{BH}}>3.6 \times 10^{9} M_{\odot}\right.$ for a non-spinning black hole $)$ would produce an accretion disk too cold to photoionize the BELR. With very massive SMBHs, one expects all emission lines to be weak and broad. Another way to form an intrinsically soft continuum is through Super-Eddington accretion, as has been proposed for the nearby $(z=0.19)$ narrow-line Seyfert 1 galaxy PHL 1811, which has exceptionally weak high-ionization lines (i.e., $W_{r}[\mathrm{C}$ IV $]=6.6 \AA$ ) and is intrinsically X-ray weak (Leighly et al. 2007a, 2007b). Super-Eddington accretion could produce softer ionizing continua through less efficient Xray production, perhaps related to a smaller or quenched X-ray corona, or X-ray photons could be advected into the black hole. Regardless of the exact physical reason, the effect is that superEddington accretion could sometimes be associated with a more narrow, UV-peaked continuum that does not emit enough high-energy photons to form high-ionization potential species (like $\mathrm{C}$ IV); however, lower-ionization species (e.g., $\mathrm{H} \alpha, \mathrm{H} \beta$, Mg II) would still be normal (Leighly et al. 2007a, 2007b).

A final mechanism proposed by Wu et al. (2011) invokes an "X-ray shielding" gas that is located interior to the BELR. Wu et al. (2011) examined the X-ray properties of an optically selected sample of $10 z \sim 2.2$ SDSS quasars that have similar rest-frame UV spectral properties to PHL 1811 (namely, weak and blueshifted C IV, and strong UV Fe emission; Leighly et al. 2007a). Compared to the parent quasar population, their PHL 1811 analogs display lower average X-ray to UV luminosity ratios (by a factor of 13) and harder average Xray spectra. To explain these observations, Wu et al. (2011) propose that an unusually large amount of X-ray absorbing gas exposes the BELR to a softer than typical continuum. Wu et al. (2011) then suggest that their PHL 1811 analogs could be a subset of WLQs, oriented such that the X-ray continuum is viewed through a column of this shielding gas; an X-ray normal WLQ would be observed at lower inclinations (also see $\mathrm{Wu}$ et al. 2012, for further observational support for this idea). The ratios of the strengths of high- to low-ionization emission lines is expected to be similar in both X-ray normal and weak objects, regardless of orientation, and we generally might expect high-ionization lines to be weaker than low-ionization lines.

Differentiating between the above scenarios requires multiwavelength observations to constrain the SED, and also broad spectral coverage in order to compare the relative strengths of high- and low-ionization emission lines. The X-ray properties of WLQs are discussed in another publication (Luo et al. 2015; also see Shemmer et al. 2009; Wu et al. 2011, 2012). Here, we focus on the UV-optical spectral properties of WLQs. Unfortunately, the existing SDSS spectra of WLQs provides 
Table 1

X-shooter Observation Log

\begin{tabular}{|c|c|c|c|c|c|c|c|c|c|}
\hline \multirow{2}{*}{$\begin{array}{l}\text { Source Name } \\
(\text { SDSS J) } \\
\end{array}$} & \multirow[b]{2}{*}{$z^{\mathrm{a}}$} & \multirow[b]{2}{*}{$z_{\text {sys }}^{\mathrm{b}}$} & \multirow[b]{2}{*}{$\alpha_{\lambda}{ }^{\mathrm{c}}$} & \multirow{2}{*}{$\begin{array}{c}i_{\mathrm{psf}}^{\mathrm{d}} \\
(\mathrm{mag}) \\
\end{array}$} & \multirow{2}{*}{$\begin{array}{c}M_{i}^{\mathrm{e}} \\
(\mathrm{mag}) \\
\end{array}$} & \multirow[b]{2}{*}{ Obs. Date } & \multicolumn{3}{|c|}{ Exp. Time $(\min )$} \\
\hline & & & & & & & UVB & VIS & NIR \\
\hline $083650.86+142539.0$ & 1.750 & 1.749 & -1.1 & 18.72 & -26.69 & $2012 \mathrm{Feb} 25$ & 38 & 40 & 40 \\
\hline $094533.98+100950.1$ & 1.671 & 1.683 & -1.1 & 17.44 & -27.80 & 2011 Dec 21 & 20 & 20 & 20 \\
\hline $132138.86+010846.3$ & 1.423 & 1.422 & -1.7 & 18.80 & -26.08 & 2013 Mar 4 & 38 & 40 & 40 \\
\hline $133222.62+034739.9^{f}$ & 1.447 & 1.442 & -0.7 & 17.94 & -26.97 & 2013 Mar 3 & 20 & 22 & 20 \\
\hline $141141.96+140233.9$ & 1.753 & 1.754 & -1.8 & 18.53 & -26.84 & 2013 Feb 12 & 38 & 40 & 40 \\
\hline
\end{tabular}

Notes.

${ }^{a}$ Redshift obtained from Hewett \& Wild (2010).

${ }^{\mathrm{b}}$ Systemic redshift (see Section 3.1).

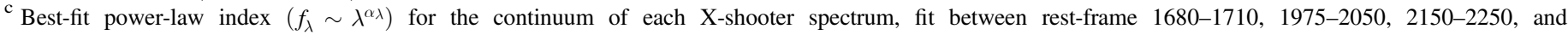
4010-4050 ̊̊ (see Section 3.2).

${ }^{\mathrm{d}}$ SDSS PSF $i$-band magnitude from Schneider et al. (2010).

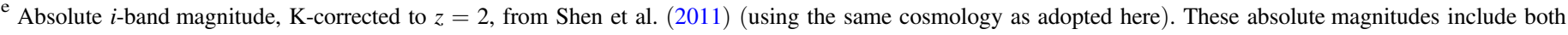

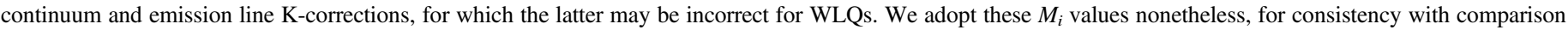
quasar samples for which we also utilize measurements from Shen et al. (2011).

${ }^{\mathrm{f}}$ A known lensed quasar; see Morokuma et al. (2007) and Section 2.2.1.

too narrow of a spectral window to place meaningful constraints on the above models, and near-infrared (NIR) spectroscopy is required to study the rest-frame optical. We therefore undertook a campaign with the X-shooter spectrograph (Vernet et al. 2011) on the Very Large Telescope (VLT), targeting a subset of seven WLQs at $1.4 \lesssim z \lesssim 1.7$. Prior to these X-shooter observations, only two other SDSS WLQs (both at $z \sim 3.5$; Shemmer et al. 2010), and one PHL 1811 analog $(z \sim 2.2$; Wu et al. 2011) had rest-frame optical constraints via NIR spectroscopy. In Section 2 we describe our X-shooter observations and data reduction. Spectral fits to emission lines are described in Section 3, and samples of comparison quasars are assembled in Section 4. We then present our results in Section 5, which are discussed in Section 6. Throughout, we adopt $H_{0}=70 \mathrm{~km} \mathrm{~s}^{-1} \mathrm{Mpc}^{-1}$, $\Omega_{\Lambda}=0.7, \Omega_{M}=0.3$. We define radio-quiet quasars as having a radio-loudness parameter $R<10$, and error bars are reported at the $68 \%$ confidence level, unless stated otherwise.

\section{X-SHOOTER OBSERVATIONS}

\subsection{Sample Selection}

We focus on WLQs at moderate redshift $(z \sim 1.5)$ here, a redshift where $\mathrm{X}$-shooter covers a large range of emission lines (spanning $\mathrm{C}$ IV through $\mathrm{H} \alpha$ ). We select targets from the lists of radio-quiet, weak-featured quasars in Table 6 of P10 (86 objects) and Table 5 of Collinge et al. 2005 (27 objects). These catalogs include quasars that pass optical spectroscopic criteria to be classified as BL Lac objects, namely, that all line emission have $W_{r}<5 \AA$, and the $4000 \AA$ break is smaller than $40 \%$ if present (see P10, for details). To select X-shooter targets, we restrict the above lists to sources with declinations $\delta \lesssim 20^{\circ}$ (to be visible to the VLT), we require targets to have a reliable redshift (from weak emission features in their SDSS spectra, typically $\mathrm{Mg}$ II, and sometimes $\mathrm{C}_{\mathrm{III}}$ ] and $\mathrm{C}$ IV) in order to avoid any contamination from stars, and to ensure that the $\mathrm{H} \beta$ line will fall in an NIR atmospheric transmission window, and we require sufficient signal-to-noise ratio $(\mathrm{S} / \mathrm{N})$ in $<1 \mathrm{hr}$ observing blocks. These cuts ween our target list to $\sim 15$ sources.
For observations, we select six targets $(1.4<z<1.7)$ with SDSS spectra that are representative of the larger $\sim 15$ object sample (in terms of relative line strengths and continuum shapes). We also include the $z=1.7$ WLQ SDSS J094533.98 +100950.1 (hereafter SDSS J0945), which was discovered serendipitously by Hryniewicz et al. (2010). We include SDSS J0945 because it has slightly stronger $\mathrm{Mg}$ II emission than the other targets, and it therefore probes a different part of WLQ parameter space (also see, e.g., Czerny et al. 2011; Laor \& Davis 2011 for constraints this particular source has placed on quasar accretion disks). Observations of these seven WLQs were taken over two observing seasons (program IDs 088.B0355 and 090.B-0438; PI Plotkin), and a summary of the observations is provided in Table 1. Throughout the text, we refer to each source by its SDSS designation truncated to the first four digits.

\subsection{Observations and Data Reduction}

Similar instrument configurations and observing strategies were employed for all seven observations. X-shooter splits the incoming light beam into three segments or "arms" (termed UVB, VIS, and NIR) using two dichroics at 560 and $1024 \mathrm{~nm}$, and the light is fed into three independently operated spectrographs. We used a $1.0 \times 11^{\prime \prime} 0$ slit for the UVB arm and $0.9 \times 11^{\prime} .0$ slits for the VIS and NIR arms, providing spectral resolutions of $R \sim 4350,7450$, and 5300 in the UVB, VIS, and NIR arms, respectively. The detectors were binned by 2 pixels along the dispersion direction for the UVB and VIS arms, and no binning was used for the NIR arm. We acquired four exposures for each target, nodding the target along the slit in an ABBA pattern (4."5 separation between positions). We observed SDSS J0945 and SDSS J1332 for 20 minutes each, and each of the other five targets were observed for $\sim 40$ minutes (see Table 1). To improve $\mathrm{S} / \mathrm{N}$ in the NIR arm, the four observations from 2013 were taken with the K-band blocking filter in place (the blocking filter was not available at the time of the other three observations). For telluric corrections, an A0V star was observed before or after each 

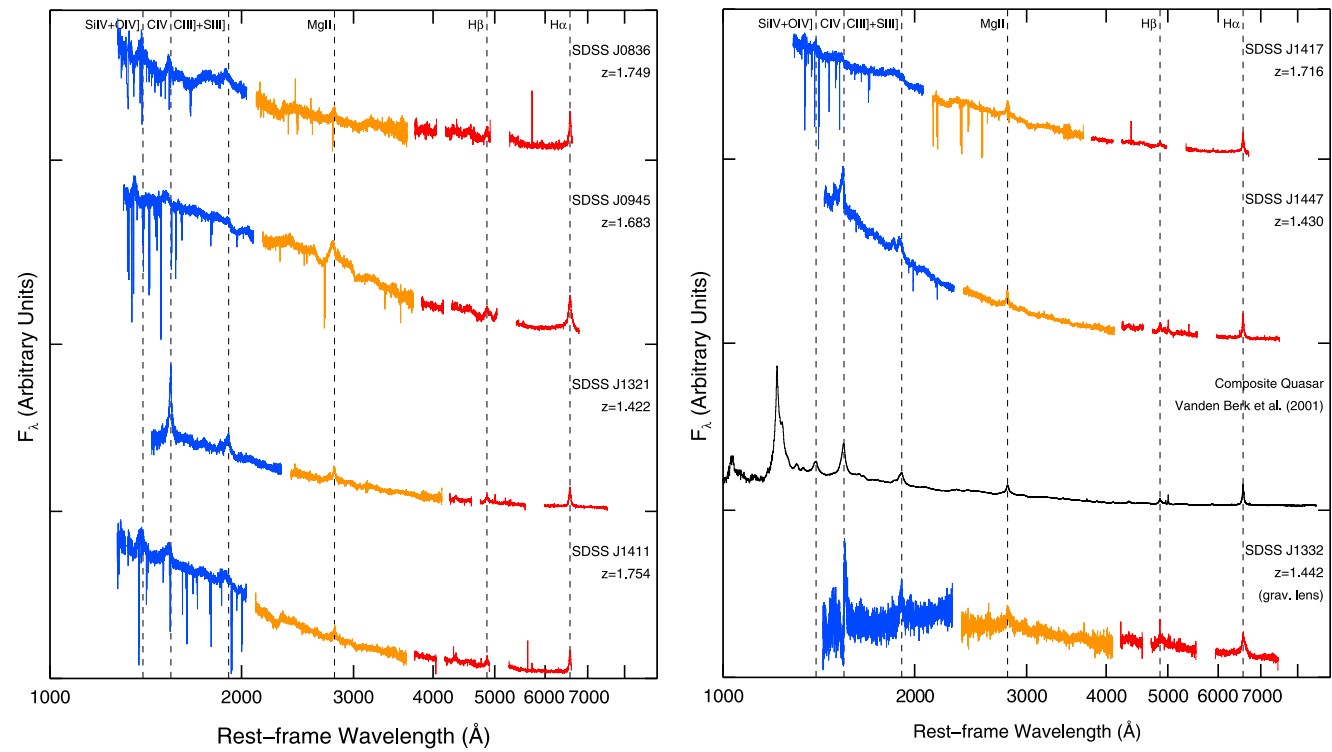

Figure 1. Full X-shoter spectra. The UVB, VIS, and NIR arms are shown in blue, orange, and red, respectively. Resolution elements are $\sim 2-3 \AA$ in each arm. Positions of prominent emission lines are indicated. (Cont'd) The bottom two spectra are the composite SDSS quasar spectrum from Vanden Berk et al. (2001) for comparison, and SDSS J1332 (for completeness) which is a gravitationally lensed quasar.

WLQ at a similar airmass, with the same slit and instrument configuration. A spectrophotometric standard star was observed for flux calibration at the beginning or end of each night using a $5^{\prime \prime}$ slit (the flux standard was taken on the following night for SDSS J1321 and SDSS J1417). Each X-shooter arm is reduced individually using the $\mathrm{X}$-shoter pipeline (Modigliani et al. 2010), following standard procedures that are described in detail in the Appendix. The final flux calibrated spectra (including telluric corrections, and corrections for Galactic extinction) are presented in Figure 1.

Absolute flux calibration of echelle spectroscopy is difficult, especially for an instrument like X-shooter with a very broad spectral range (see, e.g., Pita et al. 2014, for a recent discussion specific to X-shooter). We crudely estimate the accuracy of our flux calibration by comparing the luminosity at $5100 \AA$, $L_{5100}=\left(\nu L_{\nu}\right)_{5100}$, in our flux calibrated X-shooter spectra to $L_{5100}$ inferred from SDSS photometry (extrapolating from the psf $i$-band magnitude, and assuming a continuum following $f_{\nu}$ $\propto \nu^{-0.5}$; e.g., Vanden Berk et al. 2001). The X-shooter luminosity at $5100 \AA$ is typically $20 \%-50 \%$ lower than in the SDSS, except for SDSS J0836 where X-shooter is brighter by $\sim 10 \%$. We suspect that variability could contribute up to $\sim 15 \%$ of the difference in flux, based upon a comparison of the $i$-band psf magnitude from the SDSS photometric epoch to the synthetic $i$-band magnitude from the SDSS spectroscopic epoch for each source. Of course, we do not expect variability to systematically bias the flux calibration in the same direction for all sources. Regardless, we suspect that our X-shooter flux calibration is systematically biased toward lower values for most sources (although the magnitude of the bias is not welldetermined). This bias could in turn influence measurements of line luminosities, black hole masses, and Eddington ratio estimates. We stress, however, that $W_{r}$ and FWHM measurements are not affected by uncertainties in the flux calibration.

\subsubsection{SDSS J133222.62+034739.9}

$\operatorname{SDSSJ1332}(z=1.4)$ is a known, doubly imaged gravitationally lensed quasar (Morokuma et al. 2007). Even though there is currently no compelling evidence that lensing is related to the WLQ phenomenon (see, e.g., Shemmer et al. 2006; DS09), SDSS J1332 provides an interesting test case to further investigate any potential relationship between lensing and WLQs. For example, one may expect extinction from the foreground galaxy, and/or different magnifications of the quasar continuum compared to the BELR due to their different physical sizes. The SDSS spectrum of SDSS J1332 does not appear remarkably different from other SDSS WLQs. However, X-shooter provides bluer wavelength coverage than the SDSS, and from X-shooter it is clear that the UV continuum of SDSS J1332 is redder than the other targets, and an absorption trough is seen blueward of C IV. It is not obvious if the different continuum is predominantly intrinsic to the quasar (i.e., associated dust obscuration) or related to the gravitational lens. Regardless, this source illustrates that lensing unlikely contributes to the unusual spectral features of WLQs, since a quasar known to be lensed displays different properties from the rest of the population. We include the X-shooter spectrum of SDSS J1332 in Figure 1 and Table 1 for completeness. However, we exclude SDSS J1332 from further analysis to avoid any possible systematics due to extrinsic effects related to the lens.

\section{SPECTRAL ANALYSIS}

\subsection{Systemic Redshifts}

Prior to these X-shooter spectra, our targets' redshifts were estimated from rest-frame UV emission lines in their SDSS spectra. The SDSS-derived redshifts are listed in Table 1, as taken from Hewett \& Wild (2010). UV emission lines can be susceptible to blueshifts (relative to their laboratory wavelengths) due to winds or other non-viralized motion. From the $\mathrm{X}$-shooter NIR spectra, we derive systemic redshifts $\left(z_{\text {sys }}\right)$ from lower-ionization emission lines, which have profiles that are expected to be dominated be virialized motion. Systemic redshifts are determined, in order of preference, from the peak of narrow [O III] (which is firmly detected only for 
SDSS J1321) or from the average of the peaks of broad $\mathrm{H} \alpha$ and $\mathrm{H} \beta$. We estimate that we can determine the wavelength of the peak line flux density to within one resolution element $(\sim 3 \AA$ in the NIR), so that typically $\sigma_{z_{\text {sys }}} \lesssim 0.0006$. The new systemic redshifts are similar to the SDSS redshifts from Hewett \& Wild (2010) except for two sources, where the new systemic redshifts are slightly larger by $\Delta z=0.012$ (SDSS J0945) and 0.006 (SDSS J1417). The X-shooter derived systemic redshifts are listed in Table 1 and are adopted as the redshift for each source throughout the rest of the text.

\subsection{Continuum Shape}

We fit a power law $\left(f_{\lambda} \sim \lambda^{\alpha_{\lambda}}\right)$ to each $\mathrm{X}$-shooter spectrum in order to constrain the shape of the UV continuum. The power law is fit between the following (rest-frame) wavelength regions, 1680-1710, 1975-2050, 2150-2250, and $4010-4050 \AA$, by using a $\chi^{2}$ minimization routine (the fit is performed to all measured flux densities within each spectral window, weighted by the uncertainty on each measurement). The above spectral windows are relatively free from contamination from emission lines (including blended iron emission), they are common among all seven $\mathrm{X}$-shooter targets (given their redshifts), and they provide a wide dynamic range. The best-fit spectral indices are given in Table 1 and, excluding SDSS J1332, they are typical of other SDSS quasars (which have $\alpha_{\lambda} \approx-1.56$; Vanden Berk et al. 2001).

\subsection{Line Fitting}

For each of the six WLQs, we perform spectral fits to the $\mathrm{H} \alpha, \mathrm{H} \beta, \mathrm{Mg}$ II, and $\mathrm{C}$ IV complexes, fitting each complex separately by following a procedure similar to Trakhtenbrot \& Netzer (2012). These are among the strongest emission lines in quasars, and the ones that can be measured most accurately given the $\mathrm{S} / \mathrm{N}$ of our data. Our spectral model includes a local linear continuum, (up to three) Gaussians to model each emission line, and templates for broadened $\mathrm{Fe}$ II and Fe III emission (based on Boroson \& Green 1992 in the optical and Vestergaard \& Wilkes 2001 in the UV; see Appendix C of Trakhtenbrot \& Netzer 2012 for details). Details on the spectral fitting (including how we associate uncertainties to best-fit parameters) are provided in the Appendix. For the $\mathrm{H} \beta$ complex of SDSS J1321 and SDSS J1447, we include narrow Gaussians to model the $[\mathrm{O}$ III $] \lambda 4959,5007$ emission lines. These are the only two targets with sufficient spectral coverage to include [O III] profiles in the spectral modeling (due to their relatively lower redshifts). We also attempted to include other narrow emission line species that fall within each complex, but doing so did not improve the quality of any other fits (see Appendix). The best-fit models to each complex are shown in Figures 2 and 3 , and the corresponding spectral properties (i.e., $W_{r}$, FWHM, and line luminosities) are presented in Tables $2-4$.

\section{SAMPLES OF COMPARISON QUASARS}

\subsection{Additional WLQs with NIR Spectroscopy}

Only three other SDSS WLQs have NIR spectroscopy, all of which are at higher redshift than our X-shooter targets. These sources include SDSS J114153.34+021924.3 (hereafter SDSS $\mathrm{J} 1141 ; z=3.55$ ) and SDSS J123743.08+630144.9 (hereafter SDSS J1237; $z=3.49$ ) presented by Shemmer et al. (2010), and SDSS J152156.48+520238.5 (hereafter SDSS J1521; $z=2.238)$ presented by $\mathrm{Wu}$ et al. (2011). We note that $\mathrm{Wu}$ et al. (2011) identified SDSS J1521 as a PHL 1811 analog, and it is very X-ray weak (by a factor of 34.5) and extremely optically luminous $\left(M_{i}=-30.19 \mathrm{mag}\right.$; Just et al. 2007).

We also consider the quasar PG $1407+265(z=0.94)$, which we consider to be the "prototype" WLQ because it displays similar properties to the WLQ population later identified by the SDSS. PG $1407+265$ was identified by McDowell et al. (1995) as having an unusual BELR, with extremely weak highionization emission lines but otherwise normal quasar properties. We also consider PHL $1811(z=0.19)$, another AGN with unusual BELR properties similar to WLQs (see Leighly et al. 2007a for a broad spectrum covering Ly $\alpha$ through $\mathrm{H} \alpha$ ).

There is no uniform data coverage among the five quasars listed above. We therefore consider only their C IV and $\mathrm{H} \beta$ equivalent widths, which are included in Table 2 for reference. These five sources generally show relatively normal low-ionization emission lines, weak high-ionization lines, and often blueshifted $\mathrm{C}$ IV emission.

\subsection{Samples of Parent Quasars}

We create samples of other quasars, in order to compare our WLQs to the parent population. We start with the SDSS Data Release 7 quasar catalog (Schneider et al. 2010) and the emission line measurements provided by Shen et al. (2011). We restrict the SDSS quasar sample to only include quasars targeted for SDSS spectroscopy using the Richards et al. (2002) algorithm, which is flux limited to $i=19.1 \mathrm{mag}$, and we also exclude all objects identified as weak-featured quasars in Collinge et al. (2005) and P10. We only include quasars with absolute $i$-band magnitudes $M_{i}>-28 \mathrm{mag}$ (K-corrected to $z=2$ ), to compare to quasars that are of similar luminosities as our X-shooter targets. We also require quasars to not be flagged as broad absorption line quasars (BALs) by Shen et al. (2011), and to not be radio-loud (as determined by Shen et al. 2011).

\subsubsection{The "Intermediate-redshift" Sample}

For comparisons to rest-frame UV emission lines, we consider the subset of the above quasars at $1.5<z<2.0$. SDSS spectra of quasars in this redshift range include both $\mathrm{C}$ IV and $\mathrm{Mg}$ II, allowing a comparison of both emission species within individual objects. We further remove quasar spectra with large fractions of bad pixels within each emission complex, requiring $>250$ and $>300$ usable pixels for $\mathrm{C}$ IV and $\mathrm{Mg}$ II, respectively. The final sample includes 10,956 quasars with $-28.00<M_{i}<-25.87 \mathrm{mag}$ (median $M_{i}=-26.6 \mathrm{mag}$ ). We refer to these quasars as the "SDSS intermediate-redshift" (SDSS-IZ) subset.

\subsubsection{The "Low-redshift" Sample}

To compare to the $\mathrm{H} \beta$ spectral regime, we assemble a subset of comparison quasars at $0.4<z<0.85$. This redshift range covers both the $\mathrm{H} \beta$ and the $\mathrm{Mg}$ II complexes. The overlap with $\mathrm{Mg}$ II is useful for comparing line ratios within individual objects, and also to investigate potential biases introduced from comparing lower-redshift (and typically less luminous) quasars to our higher-redshift X-shooter targets (i.e., by comparing $\mathrm{Mg}$ II properties between this subset and the SDSS-IZ subset). We require the $\mathrm{Mg}$ II and $\mathrm{H} \beta$ spectral complexes have $>300$ and $>150$ good pixels, leaving 8151 quasars with $-27.76<M_{i}<-22.97 \mathrm{mag}$ (median $M_{i}=-24.49 \mathrm{mag}$ ). We 

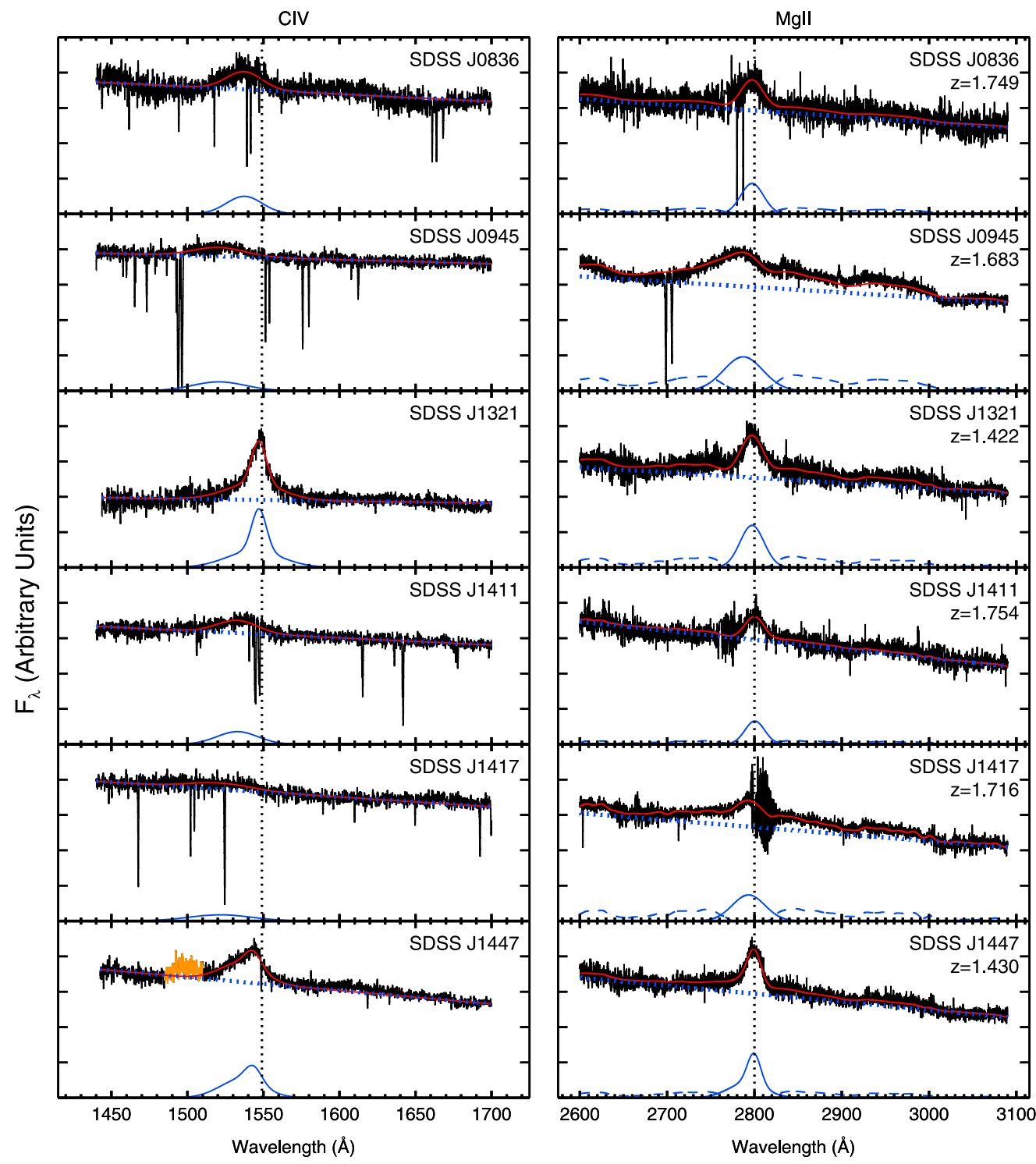

Figure 2. Expanded views of the $\mathrm{C}$ iv (left columns) and $\mathrm{Mg}$ II (right columns) complexes and their spectral fits. The same source is shown in each row. Included in each panel are the best-fit emission line profiles (solid blue line), the best-fit linear continuum (dotted blue line), and the iron continuum (dashed blue line; when required by the fit). The vertical dotted line indicates the rest-frame wavelengths of $\mathrm{C}$ IV and $\mathrm{Mg}$ II, to help visualize when those lines are blueshifted relative to the systemic redshift of each quasar. Narrow absorption line systems were masked out during the spectral fitting. The orange segment for C iv of SDSS J1447 is a calibration artifact and is not included in the spectral fit (see Appendix B.2).

refer to these quasars as the "SDSS low-redshift" (SDSS-LZ) subset.

\subsubsection{The "Small" Sample}

There are only $\sim 10^{2}$ quasars in the literature with highquality spectra covering both the rest-frame UV and optical. In turn, the above SDSS-IZ and SDSS-LZ subsets represent our best means to statistically compare our targets to the parent quasar population. However, the drawback is that we cannot compare among individual objects the full range of emission species covered by the $\mathrm{X}$-shooter spectra. To address this shortcoming, we also compile a sample of $\sim 10^{2}$ quasars from the literature that have high-quality spectral coverage of both $\mathrm{C}$ IV and $\mathrm{H} \beta$.

We start with the catalog of line measurements for 85 quasars presented by Tang et al. (2012). Their sample is based on Shang et al. (2011), and includes UV-selected Palomar-
Green (PG) quasars at $z<0.4$, UV-bright quasars at $z<0.5$ with Hubble Space Telescope UV spectroscopy, and a subset of radio-loud quasars extending out to $z \sim 1.5$. We include 27 of their $z<0.5$ quasars that are not known to be radio-loud or BAL quasars. We also include quasars from the study of Baskin \& Laor (2004), who investigated the C IV and $\mathrm{H} \beta$ properties of $81 \mathrm{PG}$ quasars at $z<0.5$. We include 41 of those quasars, after removing known radio-loud quasars, BAL quasars, and sources that also appear in Tang et al. (2012). For these 41 quasars, we adopt the $\mathrm{C}$ IV measurements presented by Baskin \& Laor (2004), and $\mathrm{H} \beta$ measured on the original optical data by Trakhtenbrot \& Netzer (2012). Finally, we also include 36 quasars from Shemmer et al. (2004) and Netzer et al. (2007), who obtained rest-frame optical spectroscopy of $z \sim$ 2.4 and 3.3 quasars. We remove known radio-loud quasars and BAL quasars from their samples, and we adopt the $\mathrm{H} \beta$ measurements calculated on this subset by Trakhtenbrot \& Netzer (2012), and C Iv measurements by Shemmer \& 

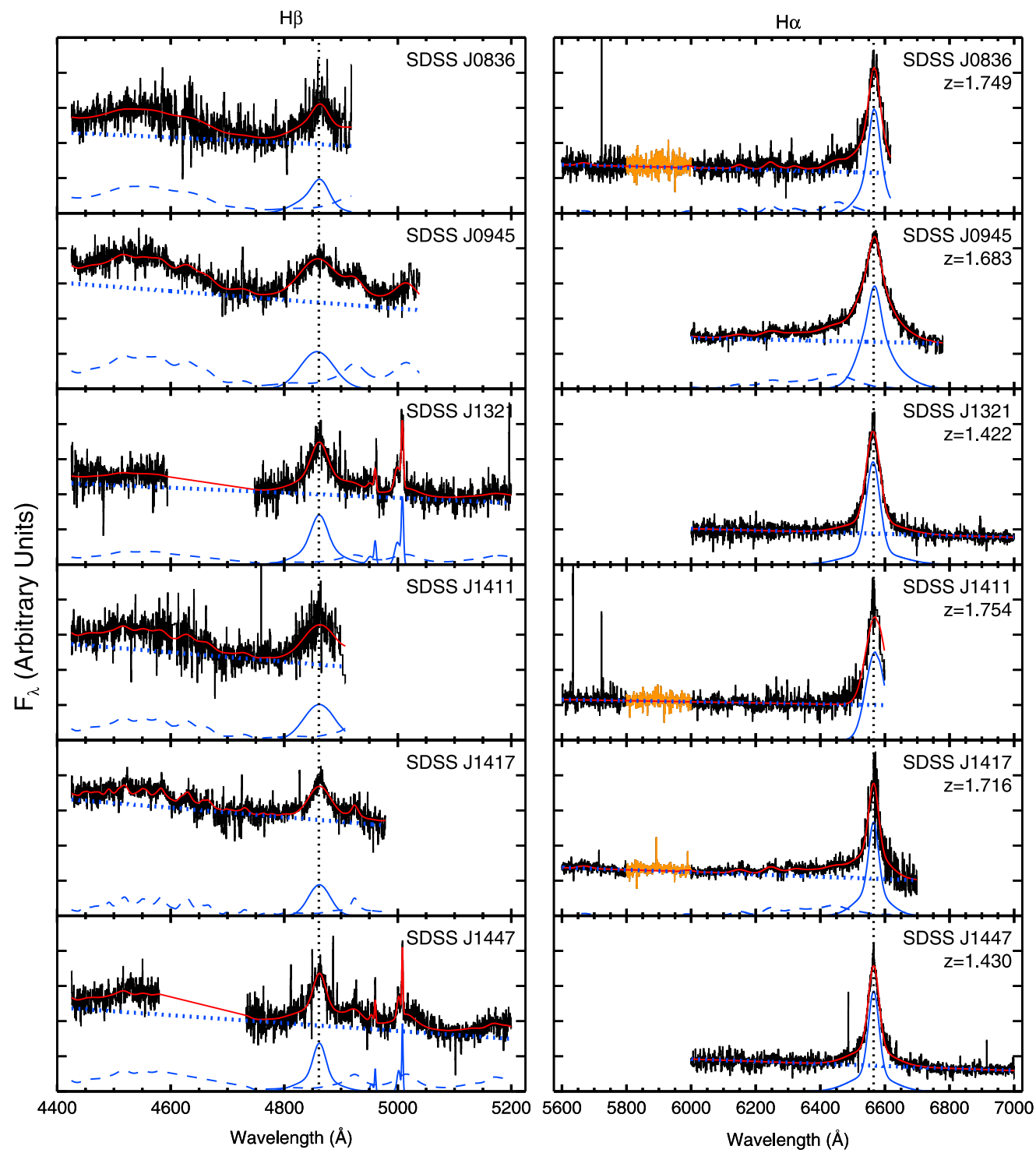

Figure 3. Expanded views of the $\mathrm{H} \beta$ (left column) and $\mathrm{H} \alpha$ (right column) complexes and their spectral fits. The format is the same as in Figure 2. Narrow Gaussians are included in the spectral fits to model [O III] for SDSS J1321 and SDSS J1447. The orange segments denote wavelengths omitted from the H $\alpha$ spectral fits for SDSS J0836, SDSS J1411, and SDSS J1417 in order to avoid potential (very weak) He i $\lambda 5877$ emission (see Section B.2).

Lieber (2015). In total, we assemble 104 quasars from the literature with $\mathrm{H} \beta$ and $\mathrm{C}$ IV coverage, which we refer to as the "small" quasar sample.

\section{RESULTS}

Here, we present the X-shooter spectral properties of our six WLQs, with physical interpretations discussed in Section 6. The NIR arm provides our first view of the rest-frame optical properties of these WLQs, and we focus on $\mathrm{H} \beta$ (not $\mathrm{H} \alpha$ ) because we do not have a statistical sample of comparison quasars covering both high-ionization emission lines and $\mathrm{H} \alpha$. Besides the extended coverage from the NIR arm, the UVB and VIS arms represent improvements over the extant SDSS spectra because of their higher spectral resolution, and because the UVB arm extends to shorter wavelengths than the SDSS.

All broad emission lines from our six WLQs have $W_{r}$ values that fall toward the weaker end of the distributions for the SDSS comparison quasars (see Figure 4). The X-shooter targets have relatively weak $\mathrm{Mg}$ II and $\mathrm{H} \beta$, but those
$W_{r}$ measures still fall within a range that is commonly displayed by the parent SDSS quasar population. On the other hand, C IV approaches a much more extreme edge of parameter space. To quantify the above statements, we follow DS09 and $\mathrm{Wu}$ et al. (2012), and we fit a lognormal profile to the $W_{r}$ distributions of $\mathrm{C}$ IV, $\mathrm{Mg}$ II, and $\mathrm{H} \beta$ for our comparison SDSS quasars. The best-fit profiles have mean $\log W_{r}=1.59 \pm 0.19, \quad 1.48 \pm 0.14$, and $1.88 \pm 0.17 \AA$ for $\mathrm{C}$ IV, $\mathrm{Mg}$ II, and $\mathrm{H} \beta$, respectively (error bars are standard deviations; the best-fit distributions for each species are similar to those found by $\mathrm{Wu}$ et al. 2012). ${ }^{16}$ As initially shown by DS09 and Wu et al. (2012), the SDSS quasar $W_{r}[\mathrm{C}$ IV $]$ distribution shows a clear excess of quasars with $W_{r}$ weaker

\footnotetext{
${ }^{16}$ We quote the $W_{r}[\mathrm{Mg}$ II] distribution for the SDSS-IZ quasars, since those quasars have luminosities more similar to our X-shooter targets compared to the SDSS-LZ subset. The $W_{r}[\mathrm{Mg}$ II $]$ distribution for the SDSS-LZ subset is similar, however, to $\log W_{r}[\mathrm{Mg} \mathrm{II}]=1.50 \pm 0.17 \AA$. Thus, there does not appear to be a large systematic bias when we compare to the SDSS-LZ subset, even though the SDSS-LZ quasars have systematically lower redshifts and luminosities.
} 
Table 2

Rest-frame Equivalent Width Measures

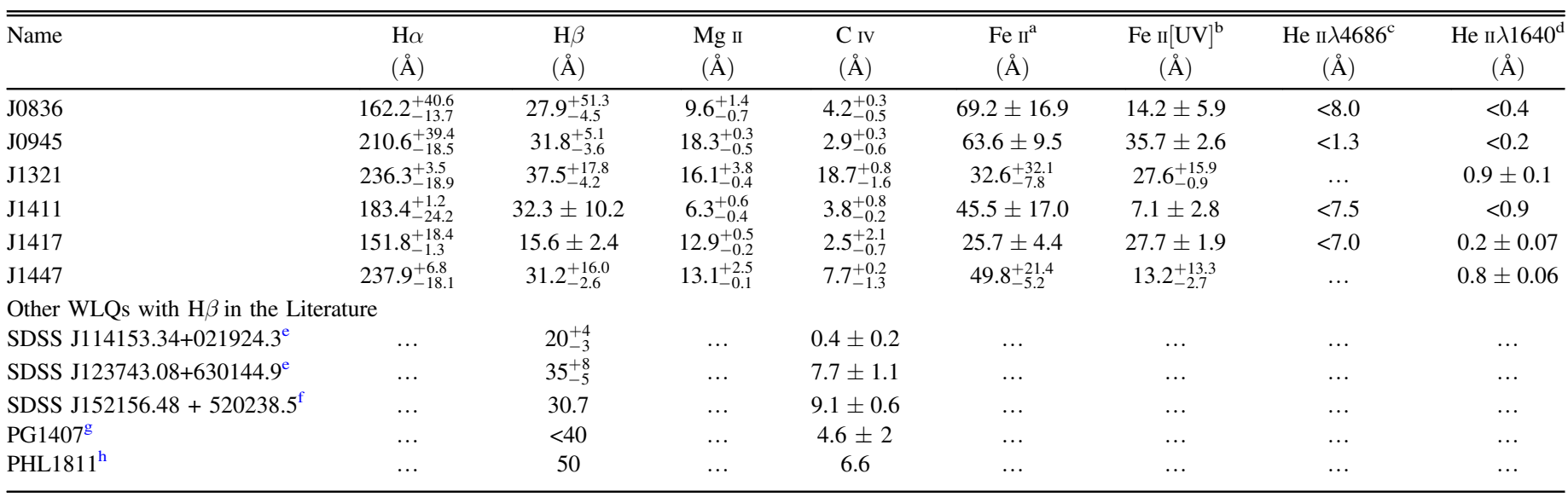

Notes.

${ }^{\text {a }}$ Equivalent width for optical Fe is calculated between $4434-4684 \AA$.

${ }^{\mathrm{b}}$ Equivalent width for UV Fe is calculated between $2250-2650 \AA$.

${ }^{\mathrm{c}} \mathrm{He}$ II $\lambda 4686$ is measured between $4665-4700 \AA$. Upper limits are quoted at the $3 \sigma$ level (see Section 6.1.1).

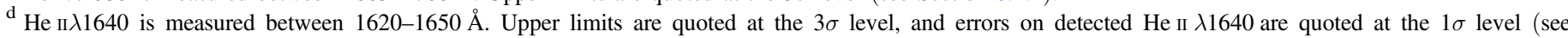
Section 6.1.1).

${ }^{\mathrm{e}} W_{r}(\mathrm{H} \beta)$ is from Shemmer et al. (2010); $W_{r}[\mathrm{C}$ IV $]$ is from DS09. Shen et al. (2011) report $W_{r}[\mathrm{C}$ IV $]=5.3 \pm 2.0 \AA$ for SDSS J123743.08+630144.9.

${ }^{\mathrm{f}} W_{r}(\mathrm{H} \beta)$ is from Wu et al. (2011); $W_{r}[\mathrm{C}$ IV $]$ is from Wu et al. (2011). Shen et al. (2011) report $W_{r}[\mathrm{C}$ IV $]=3.0 \pm 0.2 \AA$.

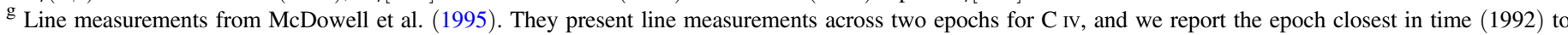
their $\mathrm{H} \beta$ observations (1994). The other $\mathrm{C}$ iv epoch has $W_{r}=3.6 \pm 2.5 \AA$.

${ }^{\mathrm{h}}$ Line measurements from Leighly et al. (2007a).

Table 3

FWHM Measures

\begin{tabular}{|c|c|c|c|c|c|c|c|}
\hline Name & $\begin{array}{c}\mathrm{H} \alpha \\
\left(\mathrm{km} \mathrm{s}^{-1}\right)\end{array}$ & $\begin{array}{r}\mathrm{Fe} \text { I }[\mathrm{H} \alpha] \\
\left(\mathrm{km} \mathrm{s}^{-1}\right)\end{array}$ & $\begin{array}{c}\mathrm{H} \beta \\
\left(\mathrm{km} \mathrm{s}^{-1}\right)\end{array}$ & $\begin{array}{c}\mathrm{Fe} \text { II }[\mathrm{H} \beta] \\
\left(\mathrm{km} \mathrm{s}^{-1}\right)\end{array}$ & $\begin{array}{c}\mathrm{Mg} \text { II } \\
\left(\mathrm{km} \mathrm{s}^{-1}\right)\end{array}$ & $\begin{array}{c}\mathrm{Fe} \text { II }[\mathrm{Mg} \mathrm{II}] \\
\left(\mathrm{km} \mathrm{s}^{-1}\right)\end{array}$ & $\begin{array}{c}\mathrm{C} \text { IV } \\
\left(\mathrm{km} \mathrm{s}^{-1}\right)\end{array}$ \\
\hline$\overline{J 0836}$ & $2298_{-412}^{+287}$ & 1575 & $2880_{-1069}^{+1877}$ & 2775 & $3284_{-210}^{+695}$ & 3850 & $\begin{array}{l}5423_{-853}^{+570} \\
\end{array}$ \\
\hline J0945 & $3368_{-50}^{+112}$ & 3525 & $4278 \pm 598$ & 1800 & $5695_{-199}^{+78}$ & 2950 & $8018_{-1730}^{+1058}$ \\
\hline J1411 & $3137_{-473}^{+282}$ & $\ldots$ & $3966 \pm 1256$ & 1475 & $2860_{-170}^{+420}$ & 1075 & $6177_{-410}^{+2210}$ \\
\hline $\mathrm{J} 1417$ & $1973_{-31}^{+140}$ & 2250 & $2784 \pm 759$ & 925 & $4717_{-51}^{+222}$ & 1375 & $9694_{-2980}^{+10305}$ \\
\hline
\end{tabular}

Table 4

Line Luminosities and Blueshifts

\begin{tabular}{|c|c|c|c|c|c|c|}
\hline Name & $\begin{array}{c}\log L[\mathrm{H} \alpha] \\
\left(\mathrm{erg} \mathrm{s}^{-1}\right)\end{array}$ & $\begin{array}{c}\log L[\mathrm{H} \beta] \\
\left(\mathrm{erg} \mathrm{s}^{-1}\right)\end{array}$ & $\begin{array}{c}\log L[\mathrm{Mg} \mathrm{II}] \\
\left(\mathrm{erg} \mathrm{s}^{-1}\right)\end{array}$ & $\begin{array}{c}\log L[\mathrm{C} \text { IV }] \\
\left(\mathrm{erg} \mathrm{s}^{-1}\right)\end{array}$ & $\begin{array}{c}\Delta v[\mathrm{Mg} \mathrm{II}] \\
\left(\mathrm{km} \mathrm{s}^{-1}\right)\end{array}$ & $\begin{array}{l}\Delta v\left[\mathrm{C}_{\mathrm{IV}}\right] \\
\left(\mathrm{km} \mathrm{s}^{-1}\right)\end{array}$ \\
\hline J0836 & $44.29_{-0.06}^{+0.21}$ & $43.71_{-0.19}^{+1.09}$ & $43.56_{-0.06}^{+0.09}$ & $43.54 \pm 0.07$ & $197 \pm 216$ & $2266 \pm 191$ \\
\hline J0945 & $44.64 \pm 0.05$ & $44.02 \pm 0.08$ & $44.29 \pm 0.02$ & $43.75 \pm 0.12$ & $\begin{array}{c}1281 \pm 183 \\
200+188\end{array}$ & $\begin{array}{c}5485 \pm 380 \\
396+189\end{array}$ \\
\hline $\mathrm{J} 1321$ & $43.89_{-0.04}^{+0.03}$ & $43.32_{-0.09}^{+0.39}$ & $43.48_{-0.02}^{+0.11}$ & $43.95_{-0.06}^{+0.26}$ & & $\begin{array}{c}396 \pm 189 \\
3142+370\end{array}$ \\
\hline J1411 & $44.03_{-0.06}^{+0.03}$ & $43.52 \pm 0.20$ & $43.34 \pm 0.06$ & $43.60_{-0.06}^{+0.16}$ & $-136 \pm 181$ & $3142_{-208}^{+370}$ \\
\hline $\mathrm{J} 1417$ & $44.15_{-0.03}^{+0.005}$ & $43.45 \pm 0.11$ & $43.88 \pm 0.02$ & $43.55_{-0.23}^{+2.38}$ & $624 \pm 180$ & $5321_{-872}^{+41178}$ \\
\hline $\mathrm{J} 1447$ & $43.99_{-0.03}^{+0.02}$ & $43.41_{-0.07}^{+0.51}$ & $43.56_{-0.02}^{+0.09}$ & $43.89_{-0.15}^{+0.50}$ & $1 \pm 185$ & $1319_{-381}^{+759}$ \\
\hline
\end{tabular}

Note. $\Delta v[\mathrm{Mg} \mathrm{II}]$ and $\Delta v[\mathrm{C}$ IV $]$ are line of sight blueshifts of the peaks of the $\mathrm{Mg}$ II and $\mathrm{C}$ IV profiles, respectively. $\Delta v$ is based on the observed wavelength of each line center (expected to be at rest-frame $2800 \AA$ for $\mathrm{Mg}$ II and $1549 \AA$ for $\mathrm{C}$ IV) compared to the systemic redshifts in Table 1 . Blueshifts are defined to be positive for approaching motions.

than the $-3 \sigma$ limit. Of the 10956 SDSS-IZ quasars, 252 have $W_{r}[\mathrm{C}$ IV $]<10.7 \AA(<-3 \sigma)$, while only 33 have $W_{r}[\mathrm{C}$ IV $]>141.3 \AA(>+3 \sigma)$. Neither $\mathrm{Mg}$ II or $\mathrm{H} \beta$ display a similar excess of quasars in their $<-3 \sigma$ tails.

The SDSS-LZ and SDSS-IZ samples might contain a small number of WLQs in their low- $W_{r}$ tails, even after removing objects identified as potential WLQs by Collinge et al. (2005) and P10. Since we do not yet have physically motivated criteria for selecting WLQs, we do not attempt to exclude additional WLQs within the comparison samples, in order to avoid also removing relatively weak-lined but otherwise "normal" quasars (see, e.g., Section 3.2 of Wu et al. 2012 for a discussion on the 

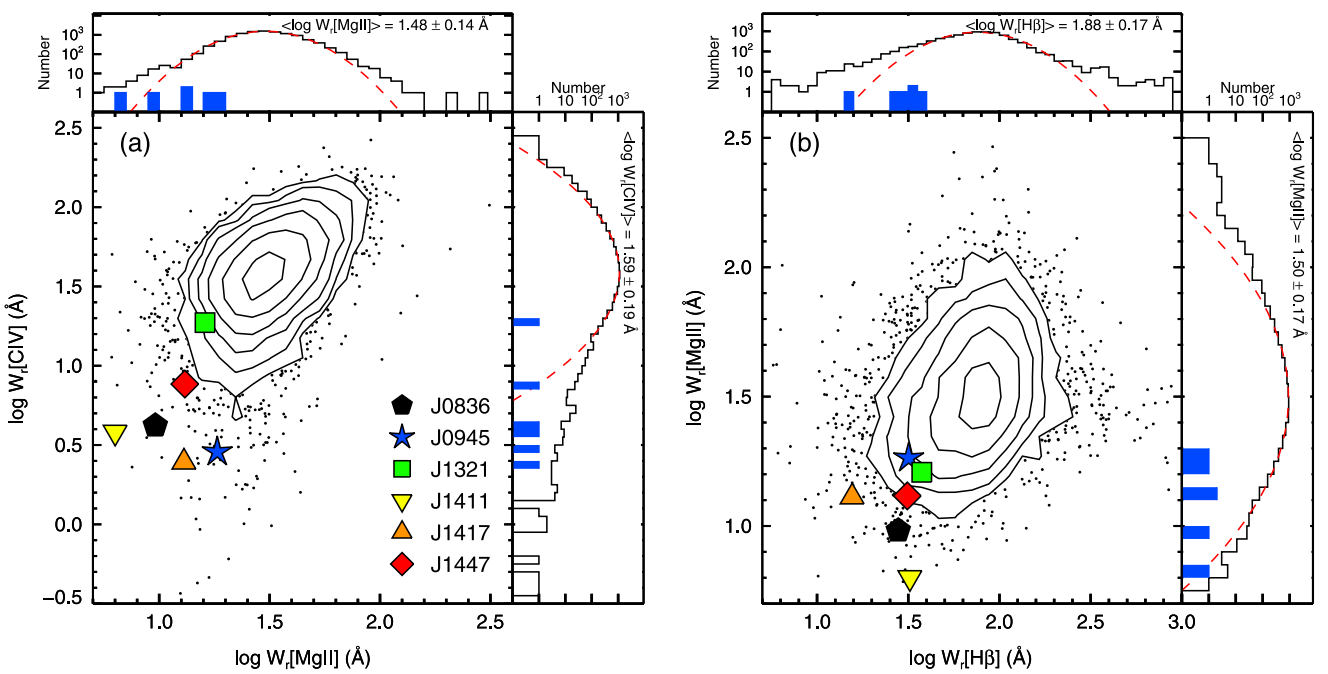

Figure 4. Logarithms of the rest-frame equivalent widths $\left(W_{r}\right)$ of our six X-shooter targets (filled symbols) compared to SDSS quasars (contours and black circles; line measurements for the comparison quasars are taken from Shen et al. 2011). (a) C IV vs. Mg II; comparison quasars are the SDSS-IZ sample. (b) Mg II vs. H $\beta$; comparison quasars are the SDSS-LZ sample. The lowest contour level in each panel denotes 10 quasars per bin $(25 \times 25$ bins along each axis), and increasing contours are logarithmically spaced by 0.35 dex; black points show individual quasars that fall below the lowest contour level. The subpanels on the top and right sides of panels (a) and (b) display histograms of the comparison quasars (black line), with the best-fit log-normal distribution plotted as a red dashed line. X-shooter WLQs are shown as filled blue histograms. Although all WLQ emission lines are weaker than typical quasars, only $\mathrm{C}$ iv is substantially weaker at the $>3 \sigma$ level (except for SDSS J1321)

(in)efficiency of WLQ selection). We stress that any unidentified WLQs in the SDSS comparison samples are too small in number to influence statistical conclusions. For example, the log-normal fit to the $W_{r}[\mathrm{C}$ IV $]$ distribution of SDSS-IZ quasars represents an accurate description of the "normal" quasars in that sample. Furthermore, the presence of any unidentified WLQs in the comparison sample would only act to bias our WLQ and comparison samples to appear more similar, thereby strengthening our conclusions that WLQs have line properties on the weaker end of the parent quasar population.

\subsection{Rest-frame Optical Properties}

Broad $\mathrm{H} \alpha$ and $\mathrm{H} \beta$ emission lines are clearly detected in the NIR spectra of all six WLQs. One source (SDSS J1417) has $W_{r}[\mathrm{H} \beta] \approx 16 \AA$, which is $4 \sigma$ weaker than the mean of the SDSS-LZ sample; all other sources display $W_{r}[\mathrm{H} \beta] \approx 28-38 \AA$, which is a rather typical value $(1.8 \sigma-2.6 \sigma$ lower than the mean). Other WLQs with $\mathrm{H} \beta$ coverage in the literature also display similar $W_{r}[\mathrm{H} \beta]$, ranging from $20-50 \AA$ (see Table 2). All six X-shooter targets also show blended Fe II emission near $\mathrm{H} \beta$. Following Boroson \& Green (1992), we define the ratio $R_{\mathrm{opt}, \mathrm{Fe} \text { II }}=W_{r}\left[\mathrm{Fe} \mathrm{II}_{\mathrm{opt}} / W_{r}[\mathrm{H} \beta]\right.$, where $W_{r}[\mathrm{Fe} \mathrm{II}]_{\mathrm{opt}}$ is measured from 4434-4684 $\AA$. For all six targets, $R_{\text {opt,Fe II }}$ lies toward the higher end of the expected distribution for the parent quasar distribution (i.e., Fe II is enhanced relative to $\mathrm{H} \beta$ ). That said, no WLQ shows an exceptionally large $R_{\text {opt,Fe II }}$ (see Figure 5). WLQs generally possess rest-frame optical properties that are consistent with other quasars that display enhanced $\mathrm{Fe}$ II emission. For example, all six X-shooter targets have $\mathrm{FWHM}[\mathrm{H} \beta] \lesssim 4000 \mathrm{~km} \mathrm{~s}^{-1}$, which is consistent with other quasars that have similar $R_{\mathrm{opt}, \mathrm{Fe}}$ II values (Boroson \& Green 1992; Sulentic et al. 2000, 2007; Shen \& Ho 2014, also see Figure 5).

To quantify the above statements, we compare the $R_{\mathrm{opt}}$ Fe ${ }_{\text {II }}$ distribution of WLQs and SDSS-LZ quasars by running Kolmogorov-Smirnov (K-S) and Mann-Whitney (M-W)

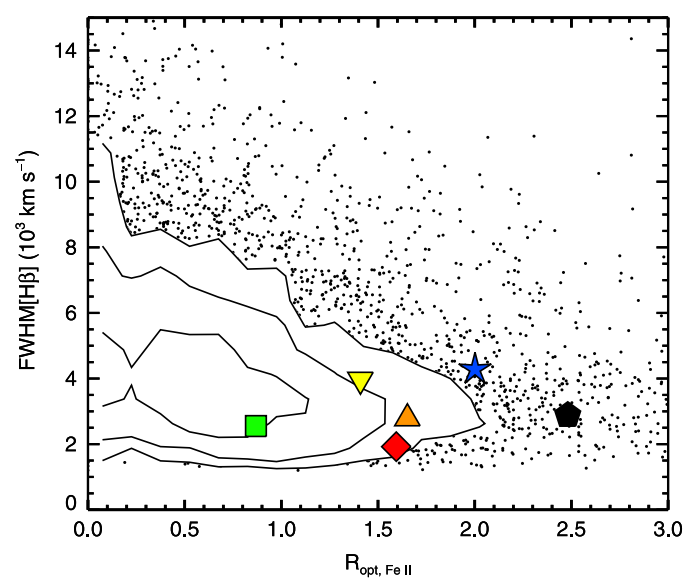

Figure 5. FWHM of $\mathrm{H} \beta$ vs. $R_{\mathrm{opt}, \mathrm{Fe} \mathrm{I}}$, the rest-frame equivalent width ratio of optical Fe II (4434-4684 $\AA$ ) to $\mathrm{H} \beta$, for the X-shooter WLQ targets (filled symbols) and the SDSS-LZ comparison quasars (contours and points). The colors and symbols shapes for the WLQs are the same as in Figure 4; contours are logarithmically spaced by 0.35 dex, with the lowest contour denoting 20 quasars per bin $(20 \times 20$ bins along each axis $)$. Our X-shooter targets generally have enhanced optical Fe II and FWHM $[\mathrm{H} \beta] \lesssim 4000 \mathrm{~km} \mathrm{~s}^{-1}$, but they do not show rest-frame optical properties atypical from other quasars with enhanced optical Fe II.

tests. The $\mathrm{K}-\mathrm{S}$ and $\mathrm{M}-\mathrm{W}$ tests are both non-parametric tests that assess if two (independent) distributions are drawn from the same parent population. The $\mathrm{K}-\mathrm{S}$ test achieves this by comparing the maximum deviation between the cumulative distribution functions of the two observed populations, while the $\mathrm{M}-\mathrm{W}$ test uses rank-ordering to compare the median values of the two distributions (e.g., Sheskin 2011). Given the small number of objects in our WLQ sample, we opt to run both tests here as alternative ways to illustrate statistical conclusions. The $R_{\text {opt,Fe II }}$ distribution of WLQs is different than that of the SDSS-LZ quasars at the $>99 \%$ level $(p=0.004$ from a K-S test; $p=0.001$ from a M-W test), indicating that WLQs as a population are indeed statistically more likely to display 
enhanced optical $\mathrm{Fe}$ II emission. We again stress that any potentially unidentified WLQs in the SDSS-LZ sample are too small in number to bias the above statistics (and any bias would only strengthen our conclusions, since the bias would force the two distributions to appear more similar).

We expect quasars with large $R_{\text {opt,Fe II }}$ to also show weak [O III] emission (e.g., Boroson \& Green 1992). Unfortunately, [O III] constraints are extremely limited for our X-shooter targets, as we only have $\left[\mathrm{O}_{\mathrm{III}}\right]$ coverage for the two targets at $z \approx 1.4$ (i.e., SDSS J1321 and SDSS J1447; the other four targets are at too high-redshift for $5007 \AA$ to fall within an atmospheric transmission window). Indeed, only SDSS J1321 with the weakest Fe II emission $\left(R_{\text {opt,Fe } \text { II }}=0.9\right)$ has a firm [O III] detection, but constraints on more objects are required to determine if WLQ [O III] emission follows the trend expected from typical quasars.

\subsection{Rest-frame UV Properties}

The bluer sensitivity of the UVB arm compared to the SDSS provides our first constraints on $\mathrm{C}$ IV for two sources (SDSS J1321 and SDSS J1447, both are at $z \sim 1.4$ and were included in the P10 sample because of their small $\left.W_{r}[\mathrm{Mg} \mathrm{II}]\right)$. These two sources display the largest $W_{r}[\mathrm{C}$ IV $]$ of all six targets. SDSS J1321 has $W_{r}[\mathrm{C}$ IV $] \approx 19 \AA$, which is only $1.7 \sigma$ weaker than the mean $W_{r}[\mathrm{C}$ IV $]$ of the SDSS-IZ comparison sample; SDSS J1447 has $W_{r}[\mathrm{C}$ IV $] \approx 8 \AA$, which is $3.7 \sigma$ weaker than the mean. Their stronger $C_{\text {IV }}$ therefore implies that weak $\mathrm{Mg}$ II does not guarantee very weak $\mathrm{C}$ IV emission (also see $\mathrm{Wu}$ et al. 2012, for a similar conclusion). It is noteworthy that both sources have $W_{r}[\mathrm{C}$ IV $]>5 \AA$, meaning that neither would have passed the P10 spectroscopic criteria for BL Lac objects if SDSS covered C IV.

The UV emission lines covered by each SDSS spectrum remain weak in each $\mathrm{X}$-shooter observation. For example, the four $\mathrm{X}$-shooter targets with $\mathrm{C}$ IV coverage in SDSS still have $W_{r}[\mathrm{C}$ IV $]<5 \AA$. We also apply our fitting routine to the SDSS spectra to measure $W_{r}[\mathrm{Mg}$ II $]$, and $\mathrm{Mg}$ II is not highly variable between the two epochs. ${ }^{17}$ The fractional change between $W_{r}\left[\mathrm{Mg}_{\mathrm{II}}\right]$ in the SDSS and X-shooter epochs are $\left(W_{r}\left[\mathrm{Mg} \mathrm{III}_{\mathrm{SDSS}}-W_{r}\left[\mathrm{Mg} \mathrm{II} \mathrm{II}_{\mathrm{Xshooter}}\right) /-W_{r}\left[\mathrm{Mg} \mathrm{II}_{\mathrm{Xshooter}}=\right.\right.\right.$ $0.41 \pm 0.18,0.05_{-0.03}^{+0.06}, 0.34 \pm 0.29,-0.14_{-0.24}^{+0.15}, 0.19 \pm 0.08$, $0.11 \pm 0.17$ for SDSS J0836, SDSS J0945, SDSS J1321, SDSS J1411, SDSS J1417, and SDSS J1447, respectively.

The new systemic redshifts from the X-shooter data also allow improved insight into the rest-frame UV properties. With updated redshifts, we can more rigorously investigate potential velocity offsets between the peaks of high- and low-ionization emission lines. Five of our targets (SDSS J0836, SDSS J0945, SDSS J1411, SDSS J1417, SDSS J1447) display strong blueshifts in the peak wavelength of their $C$ Iv lines $\left(\Delta v>1000 \mathrm{~km} \mathrm{~s}^{-1}\right.$; calculated relative to $\left.1549 \AA\right)$, suggestive of a non-virialized component to the C IV emitting BELR gas. With the new systemic redshifts from X-shooter, we find that two of these sources (SDSS J0945 and SDSS J1417) also show

\footnotetext{
17 P10 did not account for Fe emission when they performed their spectral line measurements, because it is not necessary to do so for BL Lac selection (since the beamed synchrotron jet emission dilutes the Fe continuum). As a result, P10 systematically underestimate $W_{r}[\mathrm{Mg} \mathrm{II}]$ when the continuum is not jet dominated, and we measure $W_{r}[\mathrm{Mg} \mathrm{II}]$ greater than their $5 \AA$ threshold for all six targets when accounting for $\mathrm{Fe}$ emission. The P10 measurements on $W_{r}[\mathbf{C}$ IV $]$ are not affected by this systematic, because there is not a strong $\mathrm{Fe}$ continuum near $\mathrm{C}$ IV.
}

Table 5

Virial Black Hole Masses and Eddington Ratios

\begin{tabular}{lcccc}
\hline \hline Name & $f(L)^{\mathrm{a}}$ & $\begin{array}{c}\log L_{5100}{ }^{\mathrm{b}} \\
\left(\mathrm{erg} \mathrm{s}^{-1}\right)\end{array}$ & $\begin{array}{c}\log M_{\mathrm{BH}}{ }^{\mathrm{c}} \\
\left(M_{\odot}\right)\end{array}$ & $L_{\text {bol }} / L_{\text {Edd }}{ }^{\mathrm{c}}$ \\
\hline $\mathrm{J} 0836$ & 5.96 & $45.93_{-0.23}^{+0.10}$ & $8.59_{-0.26}^{+0.64}$ & $0.87_{-0.65}^{+1.36}$ \\
$\mathrm{~J} 0945$ & 5.85 & $46.17 \pm 0.03$ & $9.05_{-0.08}^{+0.14}$ & $0.51 \pm 0.15$ \\
$\mathrm{~J} 1321$ & 6.27 & $45.41_{-0.06}^{+0.03}$ & $8.22_{-0.13}^{+0.47}$ & $0.63_{-0.37}^{+0.23}$ \\
$\mathrm{~J} 1411$ & 6.11 & $45.64 \pm 0.10$ & $8.72 \pm 0.24$ & $0.34_{-0.17}^{+0.42}$ \\
$\mathrm{~J} 1417$ & 5.97 & $45.91 \pm 0.03$ & $8.55_{-0.17}^{+0.30}$ & $0.92 \pm 0.50$ \\
$\mathrm{~J} 1447$ & 6.16 & $45.56_{-0.06}^{+0.02}$ & $8.05_{-0.06}^{+0.51}$ & $1.33_{-0.78}^{+0.17}$ \\
\hline
\end{tabular}

Notes.

${ }^{\mathrm{a}}$ Bolometric correction from Marconi et al. (2004).

b Luminosity of the power-law continuum at $5100 \AA$. The error bars are determined in the same manner as in Section B.1.

${ }^{c}$ Quoted errors on $\log M_{\mathrm{BH}}$ and $L_{\mathrm{bol}} / \mathrm{L}_{\mathrm{Edd}}$ are based on propagating the errors determined by our spectral fitting (see B.1), and they do not include systematics or other uncertainties related specifically to the virial-based scaling technique (i.e., Equations (1) and (2)).

significant $\mathrm{Mg}$ II blueshifts (calculated relative to $2800 \AA$ ) as well $\left(1281 \pm 183\right.$ and $624 \pm 180 \mathrm{~km} \mathrm{~s}^{-1}$, respectively; see Table 4). These blueshifts are discussed in Section 6.1.

\subsection{Black Hole Masses and Eddington Ratios}

We use the fits from the $\mathrm{H} \beta$ complexes to estimate virial black hole masses $\left(M_{\mathrm{BH}}\right)$ and Eddington ratios $\left(L_{\mathrm{bol}} / \mathrm{L}_{\mathrm{Edd}}\right)$. Since the optical properties from our X-shooter spectra are not atypical compared to normal quasars, we likely can obtain representative $M_{\mathrm{BH}}$ estimates from the $\mathrm{H} \beta$ line (although see caveat below). We use the luminosity of the best-fit (linear) continuum at $5100 \AA$, $L_{5100}$, and the best-fit FWHM[H $\left.\beta\right]$, along with the empirical BELR size-luminosity relation of Kaspi et al. (2005; as modified by Bentz et al. 2009):

$$
\begin{gathered}
\frac{M_{\mathrm{BH}}}{10^{6} M_{\odot}}=5.05\left[\frac{L_{5100}}{10^{44} \mathrm{erg} \mathrm{s}^{-1}}\right]^{0.5}\left[\frac{\operatorname{FWHM}(\mathrm{H} \beta)}{10^{3} \mathrm{~km} \mathrm{~s}^{-1}}\right]^{2}, \\
L_{\mathrm{bol}} / L_{\mathrm{Edd}}=0.13 f(L)\left[\frac{L_{5100}}{10^{44} \mathrm{erg} \mathrm{s}^{-1}}\right]^{0.5}\left[\frac{\operatorname{FWHM}(\mathrm{H} \beta)}{10^{3} \mathrm{~km} \mathrm{~s}^{-1}}\right]^{-2},
\end{gathered}
$$

where $f(L)$ is a luminosity-dependent bolometric correction to $L_{5100}$. We calculate $f(L)$ from Equation (21) of Marconi et al. (2004). We adopt the above relations for consistency with Shemmer et al. (2010) and $\mathrm{Wu}$ et al. (2011), who presented $\mathrm{H} \beta$ measurements for two WLQs at $z \sim 3.5$ and one PHL 1811 analog at $z \sim 2.2$, respectively. The black hole mass and Eddington ratio estimates are listed in Table 5. We find black holes masses between $\log \left(M_{\mathrm{BH}} / M_{\odot}\right) \approx 8-9$, and Eddington ratios $L_{\mathrm{bol}} / \mathrm{L}_{\mathrm{Edd}} \approx 0.3-1.3$. The $L_{\mathrm{bol}} / \mathrm{L}_{\mathrm{Edd}}$ estimates are toward the high-end of the range typically observed for $z \sim 1.4-1.7$ quasars, although we note that the uncertainties on each measurement are rather large (see Table 5).

Our six WLQs are at higher redshift than reverberation mapped quasars to which virial black hole masses are calibrated; our WLQs also generally have larger $R_{\text {opt,Fe пा }}$ and narrower $\mathrm{H} \beta$ than most reverberation mapped quasars. These caveats may introduce systematic biases into our $M_{\mathrm{BH}}$ and $L_{\text {bol }} / L_{\text {Edd }}$ estimates (Richards et al. 2011). We thus consider 


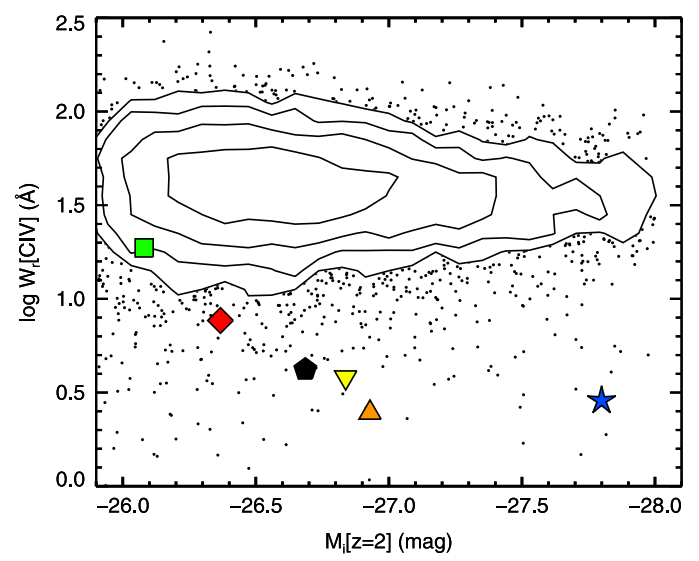

Figure 6. Logarithm of the $\mathrm{C}$ IV rest-frame equivalent width vs. $i$-band absolute magnitude for the X-shooter WLQ targets (filled symbols) and the SDSS-IZ comparison quasars (contours and points) All symbols have the same meaning as in Figure 4. The $i$-band absolute magnitudes are K-corrected to $z=2$, as reported by Shen et al. (2011) for the SDSS-IZ quasars and for our WLQs. Except for SDSS J1321 (and perhaps SDSS J1447), our WLQs have substantially weaker $\mathrm{C}$ IV rest-frame equivalent widths than expected given their continuum luminosities.

our $M_{\mathrm{BH}}$ and $L_{\mathrm{bol}} / \mathrm{L}_{\mathrm{Edd}}$ estimates to be approximate, but still useful for a qualitative analysis. We do not attempt $M_{\mathrm{BH}}$ or $L_{\text {bol }} / L_{\text {Edd }}$ estimates using either $\mathrm{C}_{\text {IV }}$ or $\mathrm{Mg}$ II because we have indications for non-virialized motion affecting those lines for some of our sources, which could bias the mass measurements (see, e.g., Baskin \& Laor 2005; Denney 2012; Trakhtenbrot \& Netzer 2012; Kratzer \& Richards 2015).

\section{DISCUSSION}

We observed with X-shooter a sample of six SDSS WLQs at moderate redshifts $(z=1.4-1.7)$. The extended spectral range provided by X-shooter compared to the SDSS allows a simultaneous comparison of the relative strengths of low- and high-ionization potential emission lines within individual objects. The X-shooter spectra definitively illustrate that when high-ionization emission lines (i.e., $\mathrm{C}$ IV) are exceptionally weak, lower-ionization lines (i.e., $\mathrm{H} \beta$ ) remain relatively normal (albeit on the weaker end of the distribution). We do not see evidence for absorption being the cause of the weak highionization emission lines in any object, since we do not observe broad absorption troughs, and the power-law continua of our $\mathrm{X}$-shooter spectra are typical of other Type 1 quasars (see Section 3.2; also see, e.g., DS09; Wu et al. 2011, 2012).

As described below, the weak high-ionization lines are unlikely to be driven by high-luminosity as might be expected from the Baldwin effect (Baldwin 1977), at least for the four $\mathrm{X}$-shooter targets with the smallest $W_{r}[\mathrm{C}$ IV $]<5 \AA$. The SDSSIZ comparison quasars span similar luminosities as our Xshooter targets $\left(-28<M_{i}<-26 \mathrm{mag}\right)$, and the SDSS-IZ quasars do not display a strong correlation between $W_{r}[\mathrm{C}$ IV $]$ and $M_{i}$ over this relatively narrow luminosity range (Figure 6); all four WLQs with $W_{r}[\mathrm{C}$ IV $]<5 \AA$ in Figure 6 have weaker $\mathrm{C}$ IV than would be expected given their luminosities (also see Shemmer \& Lieber 2015). Among the other two X-shooter targets, SDSS J1321 does not have exceptionally weak C IV for its luminosity, while SDSS J1447 is borderline.

To further illustrate the difference between high- and lowionization lines, we examine the ratio $R_{\mathrm{C} \text { IV }}=W_{r}[\mathrm{C}$ IV $] / W_{r}[\mathrm{H} \beta]$. The 104 quasars in our "small" comparison sample appear to

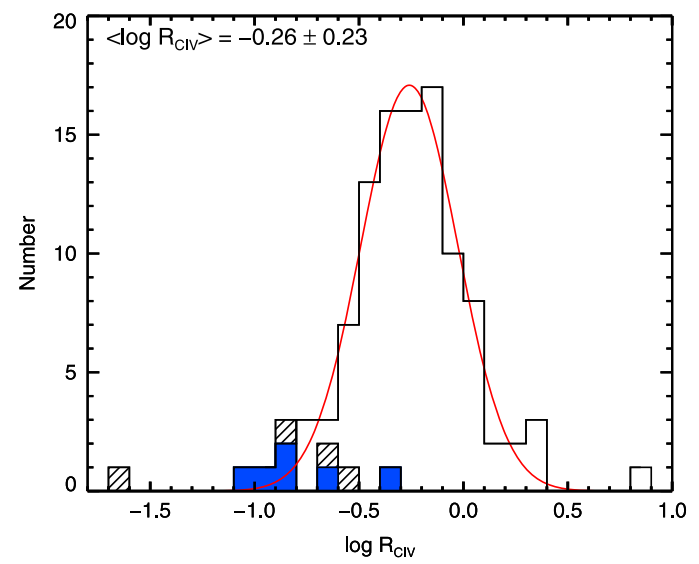

Figure 7. Distribution of $\log R_{\mathrm{C} \text { IV }}=\log \left(W_{r}[\mathrm{C} \mathrm{IV}] / W_{r}[\mathrm{H} \beta]\right)$ for the 104 object small quasar comparison sample (open histograms), with the best log-normal fit overdrawn (red curve). Filled blue histograms show the $\log R_{\mathrm{C} \text { IV }}$ distribution for our six X-shooter targets; hatched histograms show other WLQs in the literature with $\mathrm{H} \beta$ coverage (see Section 4.1), excluding PG $1407+265$ which does not have an $\mathrm{H} \beta$ detection $\left(\log R_{\mathrm{C} \text { IV }}>-0.94\right)$. WLQs populate the small $R_{\mathrm{C} \text { IV }}$ tail of the distribution, except for SDSS J1321 which has a $R_{\mathrm{C} \text { iv }}$ value typical of other quasars.

follow a log-normal distribution with $\left\langle\log R_{\mathrm{C} \text { IV }}\right\rangle=-0.26 \pm 0.23$ (quoted error is the standard deviation; see Figure 7 ). The only $\mathrm{X}$-shooter target with a

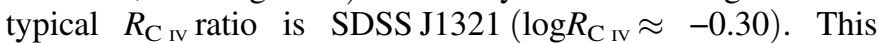
quasar also has a relatively normal $W_{r}[\mathrm{C}$ IV $]$ and negligible $\mathrm{C}$ IV blueshift. Therefore, SDSS J1321 appears to simply fall on the weak-lined tail of the normal "disk-dominated" quasar population. A relatively gas-deficient (but not completely anemic) BELR could provide an explanation for the relatively smaller (but not abnormal) $W_{r}$ measures and the typical $R_{\mathrm{C} \text { IV }}$ ratio for this source. Considering the above, we do not consider SDSS J1321 to be a WLQ.

All of the other $\mathrm{X}$-shooter targets have $R_{\mathrm{C} \text { IV }}$ ratios toward the low- $R_{\mathrm{C} \text { IV }}$ tail of the "small" quasar sample. Their $\log R_{\mathrm{C} \text { IV }}$ values are not exceptionally small (i.e., $1.5 \sigma-3.4 \sigma$ weaker). However, since our X-shooter targets were selected independent of their $\mathrm{H} \beta$ properties, we would not expect all five to populate the lower end of the $\log R_{\mathrm{C} \text { IV }}$ distribution by random chance (i.e., randomly drawing five sources that are $>1.5 \sigma$ weaker than the mean of a log-normal distribution has a probability $p \approx 10^{-6}$; a $\mathrm{K}-\mathrm{S}$ test indicates that the WLQ $R_{\mathrm{C} \text { IV }}$ distribution is different at the $p=0.003$ level, and an $\mathrm{M}-\mathrm{W}$ test finds $p=0.0002) .{ }^{18}$ Of the other WLQs/PHL 1811 analogs with $\mathrm{H} \beta$ coverage in the literature (see Section 4.1), four have $\mathrm{H} \beta$ detections. These four sources also display small $R_{\mathrm{C} \text { IV }}$ values (see hatched histograms in Figure 7), even though they also have relatively normal $W_{r}[\mathrm{H} \beta]$ values that are similar to our X-shooter targets (see Table 2).

In the simplest types of "anemic" BELR scenarios, the entire BELR would contain low amounts of gas. In that case, we generally expect WLQs to have similar $R_{\mathrm{C}_{\text {IV }}}$ values as other quasars, and we would not expect to observe preferentially weakened (and blueshifted) high-ionization lines (e.g.,

\footnotetext{
${ }^{18}$ The $\mathrm{K}-\mathrm{S}$ and $\mathrm{M}-\mathrm{W}$ tests are only meaningful if the $\mathrm{C}$ IV and $\mathrm{H} \beta$ line properties are independent, such that selecting targets based on small $W_{r}[\mathrm{C}$ IV $]$ does not bias $W_{r}[\mathrm{H} \beta]$. This assumption is likely only valid in certain circumstances, such as, e.g., the simplest "anemic" WLQ BELR scenarios. There is little concern that the "small" comparison sample includes any unidentified WLQs.
} 
McDowell et al. 1995; Dietrich et al. 2002). More complicated BELR physics must be invoked in order to explain why WLQs have small $R_{\mathrm{C} \text { rv }}$ ratios, in the context of anemic BELR scenarios. Possible explanations include a stratified BELR with a disrupted high-ionization component, or high- and lowionization gas possessing different physical properties (e.g., covering factors, densities, etc.). Regardless, it appears unlikely that low gas-content is the primary cause of the WLQ phenomenon, although it certainly could play a secondary role. A larger sample of NIR spectra of WLQs is needed to overcome small number statistics and probe the full continuum of rest-frame optical spectral properties, so that we can more rigorously compare the distributions of line ratios between WLQs and the parent quasar population.

\subsection{Soft Ionizing Continua and Disk Winds}

If the WLQ phenomenon is not primarily driven by low gas content in the BELR, then the BELR is probably exposed to an unusually soft ionizing continuum. The following discusses soft ionizing continua in the context of "disk/wind" models of the BELR (e.g., Murray et al. 1995; Elvis 2000; Leighly 2004). In these models, lower-ionization potential lines (e.g., $\mathrm{Mg}$ II, $\mathrm{H} \beta, \mathrm{H} \alpha$ ) are typically considered "disk" lines, because they are associated with gas with kinematics dominated by virialized motions (e.g., Eracleous \& Halpern 2003). High-ionization lines (like C IV) are thought of as "wind" lines, because they can show a component with non-virialized motion, often interpreted in the context of a radiatively line driven wind. Some lines can have both a disk and wind component (see, e.g., Leighly 2004 and references therein). In order to drive a wind radiatively, the balance between the number of X-ray and UV photons is critical. UV photons are required to accelerate the wind; however, a large X-ray flux will over-ionize the BELR gas, stripping too many valence electrons from the metals for the line-driving to be effective. The above scenario is consistent with some well-known correlations between quasars, specifically relations between $\mathrm{X}$-ray properties and the relative balance between disk and wind line properties (i.e., the socalled Eigenvector 1 correlates; see, e.g., Boroson \& Green 1992; Laor et al. 1997; Wills et al. 1999; Sulentic et al. 2000, 2007; Baskin \& Laor 2005; Shang et al. 2007; Gibson et al. 2009; Kruczek et al. 2011; Richards et al. 2011; Shen \& Ho 2014).

Wu et al. (2011, 2012) discuss in detail the relationship between rest-frame UV emission lines (in particular $\mathrm{C}$ Iv blueshift) and X-ray properties for WLQs/PHL 1811 analogs. The ratio of X-ray luminosity to the luminosity of the rest-frame UV continuum provides constraints on the ionizing continuum (as does the relative strengths of some UV emission species), while $\mathrm{C}$ iv blueshifts can be interpreted as a proxy for the strength of the disk wind (along the line of sight). Wu et al. $(2011,2012)$ find that PHL 1811 analogs (which were selected to have enhanced UV Fe emission, large $\mathrm{C}$ Iv blueshifts, and weak $W_{r}[\mathrm{C}$ IV $]$ ) are very X-ray weak. On the other hand, WLQs that show weaker UV Fe emission (and typically also smaller $\mathrm{C}$ iv blueshifts) are generally $\mathrm{X}$-ray normal.

To examine the properties of our $\mathrm{X}$-shooter targets in the context of the above phenomenological picture, we show our targets in the C Iv $W_{r}$-blueshift plane in Figure 8 (also see Figure 8 in Wu et al. 2012). The comparison SDSS-IZ quasars in Figure 8 have $\mathrm{C}$ Iv blueshifts calculated relative to $\mathrm{Mg}$ II, so

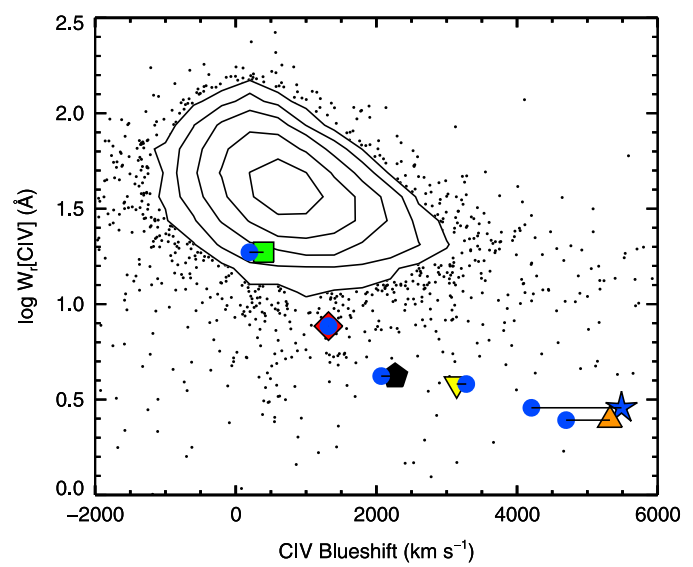

Figure 8. Logarithm of $\mathrm{C}$ IV rest-frame equivalent width vs. $\mathrm{C}$ IV blueshift (positive blueshifts denote line of sight motions moving toward the observer) for our X-shooter targets (filled symbols) and the SDSS-IZ sample (contours and small black circles). The colors and symbol shapes for the WLQs are the same as in Figure 4; contours are logarithmically spaced by 0.35 dex, with the lowest contour denoting 20 quasars $(20 \times 20$ bins along each axis $)$. For comparison to the SDSS-IZ sample, we plot the $\mathrm{C}$ IV blueshift relative to the $\mathrm{Mg}$ II line center for the X-shooter targets (filled blue circles), and also relative to the new systemic redshifts derived from the rest-frame optical spectra (other filled symbols; the symbols for each source are connected by a horizontal solid line to help guide the eye). Our WLQs (except for SDSS J1321 and perhaps SDSS J1447) appear to populate a distinct "wind-dominated" parameter space.

we present $\mathrm{C}$ iv blueshifts for our $\mathrm{X}$-shooter targets relative to both $\mathrm{Mg}$ II (blue circles) and to the X-shooter derived systemic redshift (other filled symbols). SDSS J1321 is the only target that clearly occupies a similar parameter space as typical quasars. The other five sources lie in the "wind-dominated" quadrant of Figure 8 (see, e.g., Richards et al. 2011), albeit with generally much weaker $W_{r}[\mathrm{C}$ Iv $]$ than other quasars displaying similar blueshifts. ${ }^{19}$

It is interesting that the two X-shooter targets with the strongest $\mathrm{C}$ Iv blueshifts are the only two that also display blueshifted $\mathrm{Mg}$ II emission. $\mathrm{Mg}$ II is often thought of as a disk emission line, given its relatively low ionization potential $\left(\chi_{\text {ion }} \approx 8 \mathrm{eV}\right)$. However, $\mathrm{Mg}$ II can still have a non-negligible cross section to UV radiation, and it sometimes has a wind component (e.g., Shang et al. 2007). From our X-shooter sample, it appears that a relatively strong wind is required to cause $\mathrm{Mg}$ II to display an outflowing component. We also speculate that there could be a connection between the wind strength and the amount of $\mathrm{Fe}$ II UV emission. We quantify the strength of the $\mathrm{Fe}$ II UV emission as $R_{\mathrm{uv}, \mathrm{Fe} \text { II }}=W_{r}[\mathrm{Fe} \text { II }]_{\mathrm{uv}} /$ $W_{r}[\mathrm{H} \beta]$, where $W_{r}\left[\mathrm{Fe} \mathrm{II}_{\mathrm{uv}}\right.$ is calculated from $2250-2650 \AA$, which we show relative to $\mathrm{FWHM}[\mathrm{H} \beta]$ in Figure 9. Similar to the rest-frame optical Fe II emission (i.e., $R_{\mathrm{opt}, \mathrm{Fe} \text { II }}$; Figure 5), none of the X-shooter targets displays abnormally large $R_{\mathrm{uv}}$, Fe II. However, the two WLQs showing $\mathrm{Mg}$ II blueshifts are the only ones to display relatively enhanced $R_{\mathrm{uv}, \mathrm{Fe} \text { II }}$ (compared to all five sources with $C_{\text {Iv }}$ blueshifts showing enhanced $R_{\mathrm{opt}}$, $\left.\mathrm{Fe}_{\text {II }}\right) . \mathrm{Mg}$ II blueshift is therefore a newly identified parameter that could ultimately provide additional clues on the BELR

\footnotetext{
19 There is likely a link between the FWHM $[\mathrm{H} \beta]-R_{\text {opt,Fe II }}$ plane (Figure 5) and the C Iv $W_{r}$-blueshift plane (Figure 8), in that quasars that fall in one quadrant in one plane typically map to a similar quadrant in the other plane (e.g., Sulentic et al. 2007; Richards et al. 2011). Indeed, the five X-shooter targets with large $C$ iv blueshifts $\left(>1000 \mathrm{~km} \mathrm{~s}^{-1}\right)$ also have rest-frame optical properties typical of wind-dominated quasars (i.e., large $R_{\mathrm{opt}, \mathrm{Fe} \mathrm{II}}$ and narrower FWHM $[\mathrm{H} \beta])$.
} 


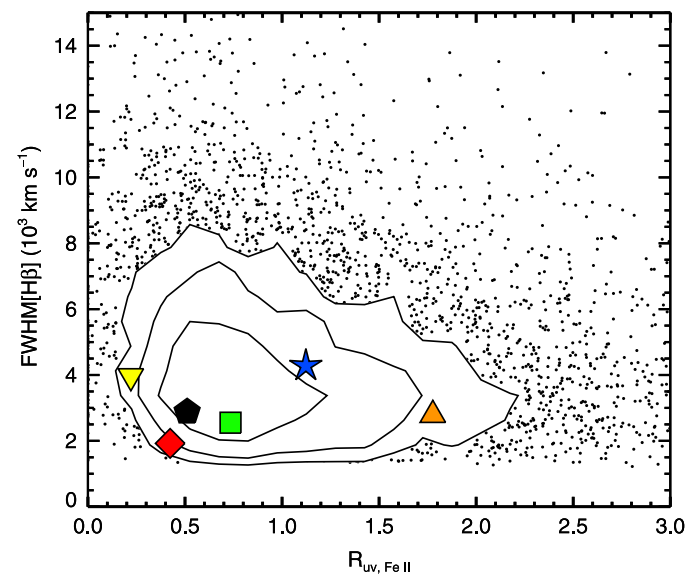

Figure 9. Similar to Figure 5, except that the $x$-axis here shows the rest-frame equivalent width ratio of UV Fe II (2250-2650 $\AA$ ) to $\mathrm{H} \beta$, compared to the SDSS-LZ quasars (all symbols have the same meaning as in Figure 5). Only the two WLQs with the strongest C IV winds (SDSS J0945 and SDSS J1417) show enhanced UV Fe II emission.

properties of WLQs (also see Luo et al. 2015 for a discussion on UV Fe II and X-ray weakness).

\subsubsection{Weak He II Emission}

The strengths of the He II lines at 1640 and $4686 \AA$ reflect the strength of the far-UV continuum at $\gtrsim 54 \mathrm{eV}$ (i.e., photon energies required to produce singly ionized $\mathrm{He}$ ), and therefore provide a means to test the soft SED hypothesis. A large $W_{r}[\mathrm{He}$ II $]$ implies a strong continuum that would overionize the BELR, thereby inhibiting the formation of strong winds (an anti-correlation between $W_{r}[\mathrm{He}$ II $]$ and disk wind velocity has indeed been observed by several studies; e.g., Leighly \& Moore 2004; Richards et al. 2011; Baskin \& Laor 2013; Bowler et al. 2014).

All six X-shooter spectra cover the He II $\lambda 1640$ line, and four spectra (excluding SDSS J1321 and SDSS J1447) cover the He II $\lambda 4686$ line in the NIR arm. We measure $W_{r}$ values for each He II line, with the $\lambda 1640$ line measured between $1620-1650 \AA$ (which we refer to as $W_{r}[\mathrm{He} \text { II }]_{\mathrm{uv}}$ ), and the $\lambda 4686$ line measured between $4665-4700 \AA\left(W_{r}[\mathrm{He} \text { II }]_{\text {opt }}\right)$. The UV and optical measurements are performed relative to the linear continua obtained from fitting the $\mathrm{C}$ IV and $\mathrm{H} \beta$ complexes in Section 3.3, respectively (and we remove the best-fit Fe II continuum prior to measuring $\left.W_{r}[\mathrm{He} \text { II }]_{\mathrm{opt}}\right)$.

All He II lines are weak and measured to have $W_{r}<1 \AA$. To assess the statistical significance of any potential He II emission, and to assign meaningful limits on non-detections, we run Monte Carlo simulations as follows. We make the null assumption that no $\mathrm{He}$ II line is present in any spectrum. We then take the best-fit spectral model for each $\mathrm{C}$ IV and $\mathrm{H} \beta$ complex, to which we add simulated (statistical) noise, such that each simulated spectrum follows $f_{\lambda, \text { sim }}=f_{\lambda, \text { model }} \pm f_{\lambda, \sigma}$. At each wavelength $\lambda, f_{\lambda, \text { sim }}$ is the simulated flux density, $f_{\lambda \text {,model }}$ is the flux density from the bestfit model, and $f_{\lambda \sigma}$, is randomly drawn from a Gaussian distribution with mean zero and a standard deviation set to the error bar on each observed flux density (these error bars were calculated during the initial data reduction in Section 2.2). We create 1000 simulated spectra for each source and complex, and we measure $W_{r}[\mathrm{He}$ II $]$ in each simulated spectrum. We take the standard deviation of each $N=1000$ distribution of
$W_{r}$ measures $\left( \pm \sigma_{\text {rms }}\right)$ to represent the $68 \%$ confidence interval for measuring a given $W_{r}$, in the case where variations in $W_{r}$ are due solely to statistical fluctuations. As expected, all simulated $W_{r}$ distributions appear to follow a normal distribution with $\left\langle W_{r}\right\rangle \approx 0$.

To consider He II to be detected in emission in our X-shooter spectra, we require $W_{r}[\mathrm{He}$ II $]>3 \sigma_{\text {rms }}$. Measured values for $W_{r}[\mathrm{He} \mathrm{II}]_{\mathrm{uv}}$ and $W_{r}[\mathrm{He} \mathrm{II}]_{\mathrm{opt}}$ are listed in Table 2 (for nondetections, we list the $3 \sigma_{\text {rms }}$ upper-limit). In the UV, only SDSS J1321 and SDSS J1447 are clearly detected, and SDSS J1417 is marginally detected. In the optical, none of the four sources with spectral coverage display detectable He II $\lambda 4686$. Given the poorer $\mathrm{S} / \mathrm{N}$ in the $\mathrm{X}$-shooter NIR arm, our limits on He II $\lambda 4686$ are not highly constraining. For "normal" non-BAL quasars with similar luminosities as our Xshooter targets, we expect $W_{r}[\mathrm{He} \text { II }]_{\text {opt }} \lesssim 5 \AA$ (Dietrich et al. 2002; a typical value may even be as small as $0.7 \AA$, according to Vanden Berk et al. 2001), while most of our Xshooter targets are only constrained to have $W_{r}[\text { He II }]_{\text {opt }}<7-8 \AA$.

In the UV, our constraints on He II $\lambda 1640$ are meaningful (except for SDSS J1411) and generally consistent with the idea that WLQs have soft ionizing continua. Bowler et al. (2014) find that a composite spectrum of $\sim 1000$ SDSS quasars at similar redshift and luminosity as our X-shooter targets has $W_{r}[\mathrm{He} \mathrm{II}]_{\mathrm{uv}} \approx 0.6 \AA$. Only two of our X-shooter targets have a larger $W_{r}[\mathrm{He} \mathrm{II}]_{\mathrm{uv}}$. However, these two objects (SDSS J1321 and SDSS J1447) are the least exotic sources within our sample, and they unlikely lie on as extreme of an edge of parameter space as the other targets: we argued earlier that SDSS J1321 is likely a relatively weak-lined but normal "disk-dominated" quasar; we discuss in Section 6.1.3 that SDSS J1447 may lie on the weak-lined tail of the normal "wind-dominated" quasar population. All three of the other Xshooter targets with meaningful limits have $W_{r}[\mathrm{He} \text { II }]_{\mathrm{uv}}<0.6 \AA$. As described earlier, these sources also display strong C Iv blueshifts, which supports the notion that WLQs are exotic versions of "wind-dominated" quasars with softer than typical ionizing continua.

\subsubsection{Is the Ionizing Continuum Intrinsically Soft?}

The relatively normal rest-frame optical properties suggest that WLQ BELRs are in an unusual ionization state, likely as a result of a soft ionizing continuum. Here, we exclude the possibility of an intrinsically soft SED due to a very massive SMBH. Laor \& Davis (2011) show that there is a critical mass of $M_{\mathrm{BH}}>3.6 \times 10^{9} M_{\odot}$ for a non-spinning black hole $(a=0)$, or $>1.4 \times 10^{10} M_{\odot}$ for a nearly maximally spinning prograde black hole $(a=0.998)$, where the accretion disk becomes too cold to emit enough high-energy photons to ionize the BELR. Taking our $\mathrm{H} \beta$-derived black hole masses at face value, no $\mathrm{X}$ shooter target (or any other WLQ with $\mathrm{H} \beta$ coverage) has a black hole mass this large (although see caveats in Section 5.3). Laor \& Davis (2011) also predict that $\mathrm{H} \beta$ should be very weak and broad (FWHM $[\mathrm{H} \beta]>8000$ and $16,000 \mathrm{~km} \mathrm{~s}^{-1}$ for $a=0$ and 0.998 , respectively), which is also not observed for any WLQ. Finally, Laor \& Davis (2011) predict that a cold accretion disk should peak at a wavelength $\lambda \gtrsim 3200 \AA$, which would imply that the continuum in the UVB arm of our Xshooter spectra should be redder than is observed (i.e., $\alpha_{\lambda}$ should be larger or even positive; X-shooter places stronger constraints on the continuum shape than SDSS because of its 
bluer sensitivity). Although very massive SMBHs are unlikely to be the explanation for our X-shooter targets, we stress that we cannot exclude the possibility that it is responsbile for weak lines in a handful of exceptional quasars (e.g., Laor \& Davis 2011 do not maintain that it must apply to every single WLQ, explicitly noting that it is unlikely to be the explanation for PG $1407+265) .^{20}$

The other two possibilities within the "soft continuum" group of WLQ models are an intrinsically soft SED due to super-Eddington accretion (Leighly et al. 2007a, 2007b), and an SED that is modified by X-ray shielding gas before it illuminates the BELR (Wu et al. 2011, 2012). If superEddington accretion is always associated with quenched X-ray emission, then the super-Eddington model cannot explain Xray normal WLQs (about half of the population; $\mathrm{Wu}$ et al. 2012). Among the X-ray weak WLQs, however, we cannot gain substantially new insight from the limited $X$ shooter sample into whether the SED is intrinsically soft or modified by shielding gas. Further distinguishing between the two scenarios will require statistically meaningful samples of WLQs with rest-frame optical spectral coverage, in parallel to ongoing efforts to observationally constrain the broadband properties (e.g., Luo et al. 2015). For example, one expects the ratio of high-versus low-ionization species (e.g., $R_{\mathrm{C} \text { Iv }}$ ) to correlate with the SED shape in the super-Eddington scenario. On the other hand, high-versus low-ionization line ratios should be less sensitive to the observed SED in the shielding gas model (since the observed X-ray weakness is determined primarily by orientation).

\subsubsection{SDSS J1447}

SDSS J1447 has only a modestly small $W_{r}[\mathrm{C}$ IV $] \approx 8 \AA$ and a relatively slow disk wind compared to the other $\mathrm{X}$-shooter targets $\left(\mathrm{C}\right.$ Iv blueshift $\approx 1300 \mathrm{~km} \mathrm{~s}^{-1}$ ). It is unclear whether or not this source is a very exotic quasar. For example, although its $W_{r}[\mathrm{C} \mathrm{IV}]$ is $\sim 4 \sigma$ weaker than the mean of the SDSS-IZ quasar distribution, its location in Figure 8 is not exceptionally different than other quasars with similar $\mathrm{C}$ Iv blueshifts. We expect higher Eddington ratio quasars to display smaller $W_{r}[\mathrm{C}$ IV $]$ (i.e., the "modified Baldwin Effect"; Baskin \& Laor 2004), and the $W_{r}[\mathrm{C}$ IV $]$ of SDSS J1447 is only $\sim 1.5 \sigma$ weaker than expected for its $L_{\text {bol }} / L_{\text {Edd }}$ (Shemmer \& Lieber 2015). There is likely overlap between the weak-lined tail of the normal (wind-dominated) quasar population with relatively high $L_{\text {bol }} / L_{\text {Edd }}$ (e.g., Baskin \& Laor 2004; Shemmer \& Lieber 2015; Netzer et al. 2004; Shen \& Ho 2014), and between the strong-lined tail of the WLQ population. SDSS J1447 seems to display properties in this gray area (see Shemmer \& Lieber 2015 for further discussion).

\subsection{Hryniewicz et al. (2010)}

There is a final possibility that could explain the relatively normal $\mathrm{H} \beta$ and the weak/blueshifted $\mathrm{C}$ IV in our $\mathrm{X}$-shooter spectra. Hryniewicz et al. (2010) propose a model where WLQs represent a short-lived phase $\left(\sim 10^{3} \mathrm{yr}\right)$ of an evolutionary sequence where black hole activity has just recently

\footnotetext{
${ }^{20}$ Recent studies suggest that luminous quasars at $z \sim 1.5-2$ are associated with high SMBH spins (Netzer \& Trakhtenbrot 2014; Trakhtenbrot 2014). This raises a possibility that some rare, potentially weak-lined objects in SDSS may represent a missing population of low-spin SMBHs. From our X-shooter results, WLQ black hole masses appear to be too small for WLQs to represent such a population.
}

been (re)activated. They propose that WLQs are essentially "adolescent" quasars, where the BELR has not yet finished forming. Low-ionization species (like $\mathrm{H} \beta$ and $\mathrm{Mg}$ II) that form close to the accretion disk should appear normal. However, the disk wind has not yet had sufficient time to populate regions farther above the disk, and higher-ionization "wind" species like $\mathrm{C}$ IV could appear exceptionally weak. We cannot effectively discriminate between adolescent quasars and soft ionizing continua from this $\mathrm{X}$-shooter program alone, because each scenario predicts similar spectral properties. Testing this scenario would require stringent constraints on WLQ evolution (i.e., more WLQs would be expected at higher redshifts) and/or the WLQ luminosity function. There are intriguing indications that WLQs are indeed more common at higher redshifts, from studies on the fraction of WLQs at $z \approx 3-6$ (DS09; Bañados et al. 2014). The systematics involved in WLQ selection at lower redshifts are not understood well enough to extend this type of work to $z<3$ at the moment. However, such systematics are starting to be addressed, between this X-shooter program and other NIR spectroscopic campaigns on $z<3$ SDSS WLQs (e.g., Shemmer et al. 2010; Wu et al. 2011), and also the recent efforts by Meusinger \& Balafkan (2014) at WLQ selection (we note, however, that the P10 and Meusinger \& Balafkan 2014 WLQ samples likely suffer from different sets of selection-based systematics, and as a result may not recover identical populations of rare quasars).

\subsection{Arguments Against Synchrotron Beaming}

Meusinger \& Balafkan (2014) recently applied machine learning techniques to the SDSS spectroscopic database to assemble a new WLQ sample, and they find that a substantial fraction of their WLQs have radio detections in FIRST (approximately one-quarter of their WLQ sample are radioloud). In light of this work, and the fact that PG 1407+265 may also have a beamed (albeit weak) relativistic jet (Blundell et al. 2003; Gallo 2006), we revisit the prospect of relativistic beaming here. A beamed jet is still highly unlikely to cause the weak BELRs for our X-shooter targets (as well as for PG 1407 +265 ), for the following reasons. First, all X-shooter targets were required to be radio-fainter, at the $>3 \sigma$ level, than the population of radio-loud SDSS BLLac objects in P10. Therefore, any radio jet must be low luminosity and/or weakly beamed. Any beamed continuum would also be highly variable, so that we would expect variable $W_{r}$ measures between the SDSS and X-shooter epochs (e.g., Ruan et al. 2014), which we do not observe. Furthermore, dilution from a relativistic jet would reduce the $W_{r}$ of all lines, inconsistent with our observations of enhanced optical Fe II (i.e., large $R_{\mathrm{opt}, \mathrm{Fe} \text { II }}$ ). Finally, the C IV blueshifts cannot be explained by dilution from a beamed jet, and the magnitude of the blueshifts (i.e., $\sim 10^{3} \mathrm{~km} \mathrm{~s}^{-1}$ ) for our X-shooter targets are uncommon for radio-loud quasars (Richards et al. 2011).

\section{CONCLUSIONS}

We presented X-shooter spectroscopy for six WLQs at moderate redshift $(z=1.4-1.7)$, allowing us to compare the properties of high-versus low-ionization emission species in individual objects. The NIR spectra from X-shooter more than double the number of WLQs with spectral coverage of the $\mathrm{H} \beta$ complex. Although all of our WLQs display relatively weak $\mathrm{H} \beta$ emission, none has a rest-frame optical spectrum that 
is abnormal compared to other quasars. With new constraints on $\mathrm{C}$ IV for two targets (which were lacking from SDSS), one target (SDSS J1321; $z=1.42$ ) appears to be a relatively weaklined but otherwise normal "disk-dominated" quasar. The other five targets have relatively narrow $\mathrm{H} \beta$ and slightly enhanced optical Fe emission, which is typical for "wind-dominated" quasars. Indeed, all five display C Iv blueshifts $>1000 \mathrm{~km} \mathrm{~s}^{-1}$. One of these quasars (SDSS J1447; $z=1.43$ ) may simply lie on the weak-lined tail of the normal, high- $L_{\mathrm{bol}} / \mathrm{L}_{\mathrm{Edd}}$ "winddominated" quasar population. The other four targets, however, have exceptionally weak $W_{r}[\mathrm{C}$ IV $]<5 \AA$ (and apparently weak He II $\lambda 1640$ ), and they appear to be bona fide WLQs. Exceptionally weak $\mathrm{C}$ IV emission therefore does not guarantee very weak emission from low-ionization lines (e.g., $\mathrm{H} \beta$ ), which is consistent with Wu et al. (2012), who concluded that it is extraordinarily difficult to select WLQs based solely on their low-ionization emission lines. In other words, WLQs appear to separate most cleanly from the "normal" quasar population via their high-ionization lines, especially $\mathrm{C}$ IV and Ly $\alpha+\mathrm{N} v$.

As we are now starting to build a respectably sized sample of WLQs with rest-frame optical spectra (six from this work, and five others in the literature), we determine that low amounts of gas in the BELR is unlikely the primary cause of the WLQ phenomenon. WLQs have relatively small $R_{\mathrm{C} \text { IV }}=W_{r}[\mathrm{C}$ IV $] /$ $W_{r}[\mathrm{H} \beta]$ line ratios, which is inconsistent with their BELRs simply having low gas content or small covering factors. Instead, either high-ionization lines have different physical properties than low-ionization lines, or WLQ BELRs are in an unusual photoionization state due to a soft SED. In the latter case, a larger sample of WLQs with rest-frame optical spectra are required to determine if the SED is intrinsically soft (as predicted if WLQs are super-Eddington accretors; Leighly et al. 2007a, 2007b) or if the SED is modified prior to illuminating the BELR (Wu et al. 2011). Our X-shooter targets are unlikely to have soft SEDs due to very massive black holes (e.g., Laor \& Davis 2011).

There is likely a connection between the shape of the ionizing continuum and the strength of a disk wind, and WLQs indeed appear to be exotic versions of "wind-dominated" quasars. Wu et al. (2011) show that WLQs with the largest C Iv blueshifts (i.e., strong winds) also tend to be X-ray weak, and they typically show strong UV Fe emission. Our two Xshooter targets with the largest C IV blueshifts (SDSS J0945 and SDSS J1417) are also the only two that show enhanced UV Fe emission, and they are the only two to show significant $\mathrm{Mg}$ II blueshifts. We thus add a new piece to the above picture, in that $\mathrm{Mg}$ II blueshift could be an additional parameter that might eventually help provide insight into WLQs.

Finally, we note that it is entirely reasonable that WLQs could be formed through multiple channels, and the WLQ population could very likely represent a heterogeneous population. More than one mechanism could contribute within individual objects as well (e.g., a single quasar could have weak lines due to a combination of a very massive black hole accreting at a super-Eddington rate, with excess shielding gas). The ultimate goal is therefore to determine if one mechanism tends to dominate over others, or if multiple mechanisms contribute at comparable levels (which could help us learn about the central engine in other types of less extreme quasars). Even with the small sample size considered in this study, we can already exclude low gas content across the entire BELR as the primary mechanism. We also identify a potential source of contamination to the Collinge et al. (2005) and P10 WLQ samples, in that some $z \approx 1.4$ SDSS WLQs may simply lie on the weak-lined tail of the normal quasar population (but not be exotic quasars in the same sense as other WLQs). These observations provide useful insight to assist in identifying lower-redshift WLQs in the future, in order to eventually build representative distributions of the line properties (e.g., $R_{\mathrm{C} \text { Iv }}$ ) to compare to X-ray properties of the WLQ population. This information will also be critical to constrain WLQ evolution, in order to use lower-redshift WLQs to further investigate indications that WLQs are more common at higher redshift (e.g., Hryniewicz et al. 2010; Liu \& Zhang 2011; Bañados et al. 2014). Understanding the physical nature of WLQs will ultimately provide new insight into the properties of quasar BELRs in general.

We thank the anonymous referee for constructive comments that improved this paper. We thank Lucas Ellebroek and Hughes Sana for advice on reducing the X-shooter spectra. W. N.B. and B.L. acknowledge support from NASA ADP grant NNX10AC99G, and Chandra X-ray Center grant GO314100X. XF acknowledges support from NSF grant AST 1107862. This research has made use of the NASA/IPAC Extragalactic Database (NED) which is operated by the Jet Propulsion Laboratory, California Institute of Technology, under contract with the National Aeronautics and Space Administration. This research has also made use of the SIMBAD database, operated at CDS, Strasbourg, France.

\section{APPENDIX A SPECTRAL REDUCTION}

Here, we describe details of the X-shooter spectral reduction. We processed the raw data using the $\mathrm{X}$-shooter pipeline (Modigliani et al. 2010), with esorex v3.10 and the Xshooter release kit v2.2.0. Each X-shooter arm is reduced individually. First, we create master bias (UVB/VIS) frames, as well as bad pixel maps for each arm. Single pinhole arc calibration frames are used to make a first guess for the wavelength solution and the centers of each order. The order centers are then traced using a single pinhole frame illuminated by a continuum lamp. Next, master flat fields are created by combining individual frames illuminated by the continuum lamp through the slit. A multi-pinhole arc frame is then used, along with the above first guess spectral model, to create a 2D map to determine the wavelength and spatial scale calibration of the detector. For the UVB arm, we also calculate the instrumental response by comparing on-sky observations of the spectrophotometric star to a flux table provided by the observatory. $^{21}$

The above calibration is applied to the individual science target frames, and to the corresponding telluric standard frames (for the VIS and NIR arms). Each individual frame is bias/dark subtracted (when relevent) and flatfielded, bad pixels are removed, and cosmic rays are rejected (following the rejection method described by van Dokkum 2001). The nodded frames are combined (using a "double-pass sky subtraction" method; see Vernet et al. 2011) to produce calibrated, background subtracted, wavelength rectified 2D spectra. Finally, 1D spectra

\footnotetext{
21 http://www.eso.org/sci/facilities/paranal/instruments/xshooter/tools/ specphot_list.html
} 
are extracted using a rectangular aperture centered on the source in the $2 \mathrm{D}$ spectra. The size of the extraction aperture is interactively adjusted to optimize the reduction for each source and arm. The UVB spectra are flux calibrated, using the instrumental response calculated from the spectrophotometric standard star, and a slit loss correction is applied based on the seeing (typically from 0. !9-1".2 at $5000 \AA$ ) and airmass (typically sec $z \sim 1.2-1.4$ ) of each observation.

For the VIS/NIR arms, we apply the telluric correction and flux calibration using the IDL routine xtellcor general from the Spextool package (Vacca et al. 2003). A telluric absorption spectrum is created by comparing the telluric standard's (merged 1D) spectrum to a high-resolution template for Vega (which is calibrated to the magnitude of the observed telluric star from SIMBAD and convolved to the X-shooter instrumental resolution). Flux-calibrated, telluric-corrected spectra are created by dividing the above model by the observed telluric standard spectrum, and then multiplying by the spectrum of the science target. Since the telluric standards were observed with the same instrument configuration as the science exposures, no additional slitloss correction is applied. Following flux calibration, all UVB, VIS, and NIR spectra are dereddened for Galactic extinction, using the Schlegel et al. (1998) maps (typical $A_{r} \sim 0.06-0.16 \mathrm{mag}$ ) and assuming a Cardelli et al. (1989) reddening curve with $R_{V}=3.1$.

\section{APPENDIX B \\ SPECTRAL LINE FITTING}

All line fitting is performed in the rest-frame. Each line complex is fit over a spectral window that is several hundred Angstroms wide. The width of the spectral window is customized for each source/complex (see Table B1). ${ }^{22}$ The local linear continuum is normalized within two $20 \AA$ bands centered on a blue wavelength $\left(\lambda_{\text {blue }}\right)$ and a red wavelength $\left(\lambda_{\text {red }}\right)$, where Fe emission is expected to be minimal. For the Fe continuum, we create a grid of templates convolved by a single Gaussian kernel spanning $925<$ FWHM $<20,000 \mathrm{~km} \mathrm{~s}^{-1}$, with the FWHM incremented in steps of $25 \mathrm{~km} \mathrm{~s}^{-1}$. The best-fitting continuum, line profile, and Fe template are chosen by stepping through each Fe template and applying a $\chi^{2}$ minimization routine. $^{23}$ In Table B1, we summarize for each source and complex the adopted values for the spectral fitting windows, $\lambda_{\text {blue, }} \lambda_{\text {red }}$, and whether an Fe template is required for each fit. Four sources (SDSS J0836, SDSS J0945, SDSS J1411, SDSS J1417) show narrow absorption features across the UVB (and sometimes VIS) arm. Prior to fitting each complex, we mask these narrow absorption features by applying a sigmaclipping algorithm.

Our initial fits include the above local linear continuum and Fe continuum, to which we add up to three broad Gaussians to represent the broad emission line in each complex (i.e., $\mathrm{H} \alpha$, $\mathrm{H} \beta, \mathrm{Mg}$ II, or $\mathrm{C}$ IV). The individual Gaussian components are not physically meaningful. They simply provide an analytic means to describe the line profiles, and only 1-2 Gaussians are typically needed to model each broad emission line (see

\footnotetext{
${ }^{22}$ For the $\mathrm{H} \alpha$ and $\mathrm{H} \beta$ complexes, the fitting window depends on the redshift of each source (and the NIR atmospheric transmission windows); the fitting window for the $\mathrm{Mg}$ II complex is largely determined by the need to obtain enough dynamic range to be able to adequately separate the Fe emission from the local linear continuum.

${ }^{23}$ Specifically, we use mpfit in IDL (Markwardt 2009), which uses a Levenberg-Marquardt technique for (weighted) nonlinear least squares fitting.
}

Table B1). Each broad Gaussian is constrained to have $1200<$ FWHM $<20,000 \mathrm{~km} \mathrm{~s}^{-1}$. We then repeat each fit and include extra Gaussian components for other emission lines that are expected to fall within each fitting window. Each line complex therefore has a minimum of five free parameters, which includes two free parameters for the linear continuum (the flux density at each of the two adopted normalization wavelengths), and three free parameters for each Gaussian component (the normalization, FWHM, and central wavelength). When an Fe continuum is included in the fit, there are two additional parameters - the normalization, and the FWHM of the Gaussian kernel used to broaden the Fe template.

Other emission lines that we model include narrow components for $\mathrm{H} \alpha, \mathrm{H} \beta, \mathrm{Mg}$ II, $\mathrm{C}_{\mathrm{IV}}$, and additional narrow emission line region species. For example, within the $\mathrm{H} \alpha$ complex, we include narrow components for the $[\mathrm{N}$ II] $\lambda \lambda 6548,6584$ and $\left[\mathrm{S}_{\mathrm{II}}\right] \lambda \lambda 6717,6731$ doublets, requiring the line flux ratio of the $\left[\mathrm{N}_{\mathrm{II}}\right]$ doublet to be 2.96 (e.g., Shen et al. 2011). Within the $\mathrm{H} \beta$ complex we include narrow components for $[\mathrm{O}$ III $] \lambda \lambda 4959,5007$, with the line flux ratio constrained to be 2.95 . Each narrow emission line is required to have the same FWHM (constrained to be $<1200 \mathrm{~km} \mathrm{~s}^{-1}$ ), and also required to have the same velocity offset from the systemic redshift. As we add additional components to each fit, the new fit is accepted if it improves the reduced $\chi^{2}$ by at least $20 \%$ (see, e.g., Hao et al. 2005). We also visually examine each fit to empirically confirm if an extra component is necessary (and that the above $20 \%$ threshold is reasonable). Ultimately, only SDSS J1321 displays firm narrow [O III] emission (and SDSS J1447 may have tentative detections). No other narrow emission lines are required for any other source/complex.

\section{B.1 Uncertainty Estimates on Emission Line Properties}

The uncertainties on best-fit line parameters are influenced by systematics, with the error budget dominated by uncertainties on the level of the continuum (i.e., there are degeneracies between the best-fit linear and Fe continuum levels and the parameters describing the line profiles). Since the uncertainties are not necessarily dominated by statistical fluctuations, we do not use the absolute value of the final reduced $\chi_{r}^{2}$ to assign a statistical confidence to the quality of each fit, nor do we trust the error bars on each best-fit parameter returned by the $\chi^{2}$ minimization routine (which are likely underestimated). We instead employ an empirical scheme to estimate uncertainties on each best-fit parameter, which implicitly accounts for (the dominant) systematic and statistical uncertainties.

We begin with the best spectral fit to each line complex, and we record the corresponding $\chi_{\text {best }}^{2}$ and degrees of freedom, $\nu_{\text {best }}$. In the following, we adopt the same number of Gaussians to model the line profile as used for the above best-fit, and we include an Fe template only if one is included in the best-fit. We then create a $51 \times 51$ grid of linear continua. Each continuum in the grid is set by a fixed flux level within the adopted $20 \AA$ continuum normalization bands, and we allow the flux level to vary through evenly spaced steps across the grid from (typically) 0.8-1.2 times the median observed flux density within each normalization band. The exact range across the grid varies with each source and complex, to ensure that we sample sufficient parameter space surrounding the best-fit continuum level determined earlier. We then systematically step through each continuum in the grid and refit each complex for each source (following the same procedure as described 
Table B1

Input Fitting Parameters

\begin{tabular}{|c|c|c|c|c|c|c|}
\hline$\overline{\text { Name }}$ & $\begin{array}{c}\text { Fitting Window } \\
(\AA)\end{array}$ & $\begin{array}{c}\lambda_{\text {blue }}{ }^{b} \\
(\AA)\end{array}$ & $\begin{array}{l}\lambda_{\text {red }}{ }^{b} \\
(\AA)\end{array}$ & Fe Template ${ }^{\mathrm{c}}$ & $\overline{N_{\text {broad }}{ }^{\mathrm{d}}}$ & 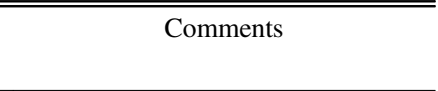 \\
\hline \multicolumn{7}{|c|}{ H $\alpha$ Spectral Complex } \\
\hline J0836 & $5600-6650^{\mathrm{e}}$ & 5650 & 6200 & BG92 & 2 & $\ldots$ \\
\hline $\mathrm{J} 1321$ & $6000-7000$ & 6200 & 6900 & $\ldots$ & 2 & $\ldots$ \\
\hline $\mathrm{J} 1411$ & $5600-6600^{\mathrm{e}}$ & 5650 & 6200 & $\ldots$ & 1 & $\ldots$ \\
\hline $\mathrm{J} 1417$ & $5600-6700^{\mathrm{e}}$ & 5650 & 6200 & BG92 & 2 & $\cdots$ \\
\hline J0836 & $4425-4920$ & 4435 & 4750 & BG92 & 2 & $\ldots$ \\
\hline J0945 & $4425-5040$ & 4435 & 4750 & BG92 & 1 & $\ldots$ \\
\hline $\mathrm{J} 1321$ & $4425-5200$ & 4435 & 5110 & BG92 & 2 & [O III] detected \\
\hline $\mathrm{J} 1411$ & $4425-4910$ & 4435 & 4750 & BG92 & 1 & $\ldots$ \\
\hline $\mathrm{J} 1417$ & $4425-4980$ & 4435 & 4750 & BG92 & 1 & $\ldots$ \\
\hline $\mathrm{J} 1447$ & $4425-5200$ & 4435 & 5110 & BG92 & 2 & [O III] tentatively detected \\
\hline $\mathrm{J} 1411$ & $2200-3090$ & 2250 & 3020 & VW01 & 1 & $\ldots$ \\
\hline $\mathrm{J} 1417$ & $2200-3090$ & 2250 & 3020 & VW01 & 1 & $\ldots$ \\
\hline $\mathrm{J} 1447$ & $2600-3090$ & 2655 & 3020 & VW01 & 2 & $\ldots$ \\
\hline \multicolumn{7}{|c|}{ C IV Spectral Complex } \\
\hline J0836 & $1440-1700$ & 1450 & 1690 & $\ldots$ & 1 & $\mathrm{C}_{\text {IV }}$ absorption doublet $(z=1.733)$ \\
\hline J0945 & $1440-1700$ & 1450 & 1690 & $\ldots$ & 1 & C IV absorption doublet $(z=1.590)^{\mathrm{f}}$ \\
\hline $\mathrm{J} 1321$ & $1440-1700$ & 1450 & 1690 & $\ldots$ & 2 & $\ldots$ \\
\hline J1411 & $1440-1700$ & 1450 & 1690 & $\ldots$ & 1 & $\ldots$ \\
\hline $\mathrm{J} 1417$ & $1440-1700$ & 1450 & 1690 & $\cdots$ & 1 & $\cdots$ \\
\hline $\mathrm{J} 1447^{\mathrm{g}}$ & $1440-1700$ & 1450 & 1690 & $\ldots$ & 2 & $\ldots$ \\
\hline
\end{tabular}

Notes.

${ }^{a}$ Rest-frame wavelength range for spectral fits.

${ }^{\mathrm{b}}$ Local linear continuum is normalized in two $20 \AA$ windows centered on $\lambda_{\text {blue }}$ and $\lambda_{\text {red }}$ (rest-frame).

${ }^{\mathrm{c}}$ Iron template used for each spectral complex. BG92: Boroson \& Green (1992), VW01: Vestergaard \& Wilkes (2001).

d Number of broad Gaussian components in best-fit.

e Rest-frame 5800-6000 Å masked from fit, to avoid potential contamination from broad He I $\lambda 5877$.

${ }^{\mathrm{f}}$ Absorption doublet also detected in SDSS spectrum by Hryniewicz et al. (2010).

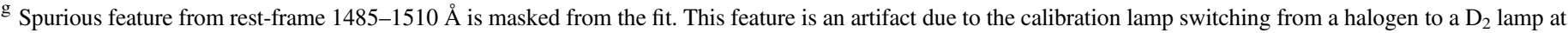
observed frame $\lambda \lesssim 3600 \AA$.

earlier, but keeping the linear continuum fixed during each fit). After each fit is performed, we calculate line properties (e.g., $\lambda_{c}$, FWHM, line flux, $W_{r}$, etc.), and we record $\chi_{i}^{2}$ (i.e., $\chi_{i}^{2}$ is for the fit using the $i$ th continuum model in the grid). Since each fit within the grid contains the same number of degrees of freedom, we consider the relative $\chi_{i}^{2}$ (i.e., $\Delta \chi_{i}^{2}=\chi_{i}^{2}-\chi_{\text {best }}^{2}$ ) across the grid to estimate uncertainties. The 68\% $(\sim 1 \sigma)$ confidence interval typically corresponds to continua providing fits with $\Delta \chi_{i}^{2} \sim 30-50$ (given $\nu \sim 1900-6500$, depending on the source and line complex). For each continuum providing a $\Delta \chi_{i}^{2}$ within the $68 \%$ confidence range, we determine the minimum and maximum value of each line property (i.e., $\lambda_{c}$, FWHM, $W_{r}$, etc.), linear continuum slope and intercept, and Fe continuum normalization (when an iron template is included in the fit). We use the minimum and maximum values of each parameter compared to the best-fit values derived earlier to determine approximate $\pm 1 \sigma$ uncertainties.

\section{B.2 Notes on Fitting Individual Objects}

SDSS J0836-Narrow absorption systems for the $\mathrm{C}_{\text {IV }} \lambda \lambda 1548,1551$ and $\mathrm{Mg}$ II $\lambda \lambda 2796,2804$ doublets are detected at $z=1.733$. When fitting the $\mathrm{H} \alpha$ complex, we mask 5800-6000 A to avoid potential broad He I $\lambda 5877$ emission blended with the continuum.

SDSS J0945-Narrow absorption systems for $\mathrm{C}_{\text {IV }} \lambda \lambda 1548,1551$ and $\mathrm{Mg}$ II $\lambda \lambda 2796,2804$ are detected at $z=1.590$. These systems were also reported by Hryniewicz et al. (2010) from the SDSS spectrum.

SDSS J1321- $[\mathrm{O}$ III $]$ is detected in the NIR arm. There is a hint of narrow $\mathrm{H} \alpha$ emission when visually examining this spectrum, but including a narrow $\mathrm{H} \alpha$ component does not improve the quality of the fit.

SDSS J1411-We mask 5800-6000 A when fitting $\mathrm{H} \alpha$ for this source. A telluric line falls near $\mathrm{Mg}$ II. The affected pixels are given less weight during the spectral fitting, but this contamination may cause an additional source of systematic uncertainty on the measurements of the $\mathrm{Mg}$ II line.

SDSS J1417-We mask 5800-6000 А when fitting $\mathrm{H} \alpha$ for this source. A telluric line also falls near $\mathrm{Mg}$ II (see notes above).

SDSS J1447- $[\mathrm{O}$ III $]$ is tentatively detected in the NIR arm. There is a hint of narrow $\mathrm{H} \alpha$ emission when visually examining this spectrum, but including a narrow 
$\mathrm{H} \alpha$ component does not improve the quality of the fit. There is also a spurious feature in the UVB arm toward the blue end of the broad C Iv emission line, near $3600 \AA$ in the observed frame. This feature is an artifact from the reduction cascade, resulting from the continuum calibration lamp switching from a halogen lamp to a $\mathrm{D}_{2}$ lamp at $\lambda \lesssim 3600 \AA$. We therefore mask rest-frame $1485-1510 \AA$ prior to fitting the C IV complex. This artifact is not present in the other targets' UVB spectra.

\section{REFERENCES}

Anderson, S. F., Fan, X., Richards, G. T., et al. 2001, AJ, 122, 503 Bañados, E., Venemans, B. P., Morganson, E., et al. 2014, AJ, 148, 14 Baldwin, J. A. 1977, ApJ, 214, 679

Baskin, A., \& Laor, A. 2004, MNRAS, 350, L31

Baskin, A., \& Laor, A. 2005, MNRAS, 356, 1029

Baskin, A., Laor, A., \& Hamann, F. 2013, MNRAS, 432, 1525

Bentz, M. C., Peterson, B. M., Netzer, H., Pogge, R. W., \& Vestergaard, M. 2009, ApJ, 697, 160

Blandford, R. D., \& Rees, M. J. 1978, in Proc. BL Lac Objects, ed. A. M. Wolfe (Pittsburgh, PA: Univ. Pittsburgh), 328

Blundell, K. M., Beasley, A. J., \& Bicknell, G. V. 2003, ApJL, 591, L103

Boroson, T. A., \& Green, R. F. 1992, ApJS, 80, 109

Bowler, R. A. A., Hewett, P. C., Allen, J. T., \& Ferland, G. J. 2014, MNRAS, 445,359

Cardelli, J. A., Clayton, G. C., \& Mathis, J. S. 1989, ApJ, 345, 245

Collinge, M. J., Strauss, M. A., Hall, P. B., et al. 2005, AJ, 129, 2542

Czerny, B., Hryniewicz, K., Nikołajuk, M., \& Sạdowski, A. 2011, MNRAS, 415, 2942

Denney, K. D. 2012, ApJ, 759, 44

Diamond-Stanic, A. M., Fan, X., Brandt, W. N., et al. 2009, ApJ, 699, 782 (DS09)

Dietrich, M., Hamann, F., Shields, J. C., et al. 2002, ApJ, 581, 912

Elitzur, M., \& Ho, L. C. 2009, ApJL, 701, L91

Elvis, M. 2000, ApJ, 545, 63

Eracleous, M., \& Halpern, J. P. 2003, ApJ, 599, 886

Fan, X., Strauss, M. A., Gunn, J. E., et al. 1999, ApJL, 526, L57

Gallo, L. C. 2006, MNRAS, 365, 960

Gibson, R. R., Jiang, L., Brandt, W. N., et al. 2009, ApJ, 692, 758

Hao, L., Strauss, M. A., Tremonti, C. A., et al. 2005, AJ, 129, 1783

Hawkins, M. R. S. 2004, A\&A, 424, 519

Heidt, J., \& Nilsson, K. 2011, A\&A, 529, A162

Hewett, P. C., \& Wild, V. 2010, MNRAS, 405, 2302

Hryniewicz, K., Czerny, B., Nikołajuk, M., \& Kuraszkiewicz, J. 2010, MNRAS, 404, 2028

Just, D. W., Brandt, W. N., Shemmer, O., et al. 2007, ApJ, 665, 1004

Kaspi, S., Maoz, D., Netzer, H., et al. 2005, ApJ, 629, 61

Kellermann, K. I., Sramek, R., Schmidt, M., Shaffer, D. B., \& Green, R. 1989, AJ, 98, 1195

Kratzer, R. M., \& Richards, G. T. 2015, AJ, 149, 61

Kruczek, N. E., Richards, G. T., Gallagher, S. C., et al. 2011, AJ, 142, 130

Lane, R. A., Shemmer, O., Diamond-Stanic, A. M., et al. 2011, ApJ, 743, 163

Laor, A., \& Davis, S. W. 2011, MNRAS, 417, 681

Laor, A., Fiore, F., Elvis, M., Wilkes, B. J., \& McDowell, J. C. 1997, ApJ, 477, 93

Leighly, K. M. 2004, ApJ, 611, 125

Leighly, K. M., Halpern, J. P., Jenkins, E. B., \& Casebeer, D. 2007a, ApJS, 173,1

Leighly, K. M., Halpern, J. P., Jenkins, E. B., et al. 2007b, ApJ, 663, 103

Leighly, K. M., \& Moore, J. R. 2004, ApJ, 611, 107
Liu, Y., \& Zhang, S. N. 2011, ApJL, 728, L44

Luo, B., Brandt, W. W., Hall, P. B., et al. 2015, ApJ, 805, 122

Marconi, A., Risaliti, G., Gilli, R., et al. 2004, MNRAS, 351, 169

Markwardt, C. B. 2009, in ASP Conf. Ser. 411, Astronomical Data Analysis Software and Systems XVIII, ed. D. A. Bohlender, D. Durand, \& P. Dowler (San Francisco, CA: ASP), 251

McDowell, J. C., Canizares, C., Elvis, M., et al. 1995, ApJ, 450, 585

Meusinger, H., \& Balafkan, N. 2014, A\&A, 568, A114

Modigliani, A., Goldoni, P., Royer, F., et al. 2010, Proc. SPIE, 7737, 28

Morokuma, T., Inada, N., Oguri, M., et al. 2007, AJ, 133, 214

Murray, N., \& Chiang, J. 1995, ApJL, 454, L105

Murray, N., Chiang, J., Grossman, S. A., \& Voit, G. M. 1995, ApJ, 451,498

Netzer, H., Lira, P., Trakhtenbrot, B., Shemmer, O., \& Cury, I. 2007, ApJ, 671,1256

Netzer, H., Shemmer, O., Maiolino, R., et al. 2004, ApJ, 614, 558

Netzer, H., \& Trakhtenbrot, B. 2014, MNRAS, 438, 672

Nicastro, F. 2000, ApJL, 530, L65

Nicastro, F., Martocchia, A., \& Matt, G. 2003, ApJL, 589, L13

Nikołajuk, M., \& Walter, R. 2012, MNRAS, 420, 2518

Peterson, B. M. 1993, PASP, 105, 247

Plotkin, R. M., Anderson, S. F., Brandt, W. N., et al. 2010a, AJ, 139, 390

Pita, S., Goldoni, P., Boisson, C., et al. 2014, A\&A, 565, A12

Plotkin, R. M., Anderson, S. F., Brandt, W. N., et al. 2010b, ApJ, 721, 562

Plotkin, R. M., Anderson, S. F., Brandt, W. N., et al. 2012, ApJL, 745, L27

Richards, G. T., Fan, X., Newberg, H. J., et al. 2002, AJ, 123, 2945

Richards, G. T., Kruczek, N. E., Gallagher, S. C., et al. 2011, AJ, 141, 167

Ruan, J. J., Anderson, S. F., Plotkin, R. M., et al. 2014, ApJ, 797, 19

Schlegel, D. J., Finkbeiner, D. P., \& Davis, M. 1998, ApJ, 500, 525

Schneider, D. P., Richards, G. T., Hall, P. B., et al. 2010, AJ, 139, 2360

Shang, Z., Wills, B. J., Wills, D., \& Brotherton, M. S. 2007, AJ, 134, 294

Shang, Z., Brotherton, M. S., Wills, B. J., et al. 2011, ApJS, 196, 2

Shemmer, O., Brandt, W. N., Anderson, S. F., et al. 2009, ApJ, 696, 580

Shemmer, O., Brandt, W. N., Schneider, D. P., et al. 2006, ApJ, 644, 86

Shemmer, O., \& Lieber, S. 2015, ApJ, 805, 124

Shemmer, O., Netzer, H., Maiolino, R., et al. 2004, ApJ, 614, 547

Shemmer, O., Trakhtenbrot, B., Anderson, S. F., et al. 2010, ApJL, 722, L152

Shen, Y., \& Ho, L. C. 2014, Natur, 513, 210

Shen, Y., Richards, G. T., Strauss, M. A., et al. 2011, ApJS, 194, 45

Sheskin, D. J. 2011, Handbook of Parametric and Nonparametric Statistical Procedures (5th ed.; Boca Raton, FL: CRC Press)

Smith, P. S., Williams, G. G., Schmidt, G. D., Diamond-Stanic, A. M., \& Means, D. L. 2007, ApJ, 663, 118

Sulentic, J. W., Bachev, R., Marziani, P., Negrete, C. A., \& Dultzin, D. 2007, ApJ, 666, 757

Sulentic, J. W., Zwitter, T., Marziani, P., \& Dultzin-Hacyan, D. 2000, ApJL, 536, L5

Tang, B., Shang, Z., Gu, Q., Brotherton, M. S., \& Runnoe, J. C. 2012, ApJS, 201, 38

Trakhtenbrot, B. 2014, ApJL, 789, L9

Trakhtenbrot, B., \& Netzer, H. 2012, MNRAS, 427, 3081

Tran, H. D., Lyke, J. E., \& Mader, J. A. 2011, ApJL, 726, L21

Trump, J. R., Impey, C. D., Kelly, B. C., et al. 2011, ApJ, 733, 60

Vacca, W. D., Cushing, M. C., \& Rayner, J. T. 2003, PASP, 115, 389

van Dokkum, P. G. 2001, PASP, 113, 1420

Vanden Berk, D. E., Richards, G. T., Bauer, A., et al. 2001, AJ, 122, 549

Vernet, J., Dekker, H., D’Odorico, S., et al. 2011, A\&A, 536, A105

Vestergaard, M., \& Wilkes, B. J. 2001, ApJS, 134, 1

Wills, B. J., Laor, A., Brotherton, M. S., et al. 1999, ApJL, 515, L53

Wu, J., Brandt, W. N., Anderson, S. F., et al. 2012, ApJ, 747, 10

Wu, J., Brandt, W. N., Hall, P. B., et al. 2011, ApJ, 736, 28

York, D. G., Adelman, J., Anderson, J. E., Jr., et al. 2000, AJ, 120, 1579 